\title{
Increases in p53 expression induce CTGF synthesis by mouse and human hepatocytes and result in liver fibrosis in mice
}

\author{
Takahiro Kodama, ${ }^{1}$ Tetsuo Takehara, ${ }^{1}$ Hayato Hikita, ${ }^{1}$ Satoshi Shimizu, ${ }^{1}$ Minoru Shigekawa, ${ }^{1}$ \\ Hinako Tsunematsu, ${ }^{1}$ Wei Li, ${ }^{1}$ Takuya Miyagi, ${ }^{1}$ Atsushi Hosui, ${ }^{1}$ Tomohide Tatsumi,, ${ }^{1}$ Hisashi Ishida, ${ }^{1}$ \\ Tatsuya Kanto,, ${ }^{1}$ Naoki Hiramatsu,, ${ }^{1}$ Satoshi Kubota, ${ }^{2}$ Masaharu Takigawa, ${ }^{2}$ Yoshito Tomimaru, ${ }^{3}$ \\ Akira Tomokuni, ${ }^{3}$ Hiroaki Nagano,, ${ }^{3}$ Yuichiro Doki, ${ }^{3}$ Masaki Mori, ${ }^{3}$ and Norio Hayashi ${ }^{4}$

\begin{abstract}
${ }^{1}$ Department of Gastroenterology and Hepatology, Osaka University Graduate School of Medicine, Suita, Osaka, Japan. ${ }^{2}$ Department of Biochemistry and Molecular Dentistry, Okayama University Graduate School of Medicine, Dentistry and Pharmaceutical Sciences, Okayama, Japan.

${ }^{3}$ Department of Surgery, Osaka University Graduate School of Medicine, Suita, Osaka, Japan. ${ }^{4}$ Kansai-Rosai Hospital, Amagasaki, Hyogo, Japan.
\end{abstract}

\begin{abstract}
The tumor suppressor p53 has been implicated in the pathogenesis of non-cancer-related conditions such as insulin resistance, cardiac failure, and early aging. In addition, accumulation of p53 has been observed in the hepatocytes of individuals with fibrotic liver diseases, but the significance of this is not known. Herein, we have mechanistically linked p53 activation in hepatocytes to liver fibrosis. Hepatocyte-specific deletion in mice of the gene encoding Mdm2, a protein that promotes p53 degradation, led to hepatocyte synthesis of connective tissue growth factor (CTGF; the hepatic fibrogenic master switch), increased hepatocyte apoptosis, and spontaneous liver fibrosis; concurrent removal of $p 53$ completely abolished this phenotype. Compared with wild-type controls, mice with hepatocyte-specific $p 53$ deletion exhibited similar levels of hepatocyte apoptosis but decreased liver fibrosis and hepatic CTGF expression in two models of liver fibrosis. The clinical significance of these data was highlighted by two observations. First, p53 upregulated CTGF in a human hepatocellular carcinoma cell line by repressing miR-17-92. Second, human liver samples showed a correlation between CTGF and p53-regulated gene expression, which were both increased in fibrotic livers. This study reveals that p53 induces CTGF expression and promotes liver fibrosis, suggesting that the P53/CTGF pathway may be a therapeutic target in the treatment of liver fibrosis.
\end{abstract}

\section{Introduction}

The tumor suppressor p53 primarily functions as a guardian of the genome, suppressing tumor development in various organs. In response to genotoxic stresses induced by DNA damage, reactive oxygen species, oncogene activation, and hypoxia, the $\mathrm{p} 53$ protein is stabilized and becomes transcriptionally active, leading to cell cycle arrest, DNA repair, and apoptosis predominantly through expression of p53-regulated genes such as $p 21, P U M A, N O X A$, and $B A X(1)$. Aside from these well-established roles, recent reports have revealed new aspects of $\mathrm{p} 53$, e.g., regulation of multiple biological functions such as glycolysis (2), anti-oxidation (3), autophagy (4), and senescence (5). It has also been demonstrated that p53 activation causes insulin resistance (6), cardiac failure (7), and early aging (5), indicating that p53 is involved even in the pathophysiology of various non-tumorous conditions via its numerous functions.

Organ fibrosis is considered to be a major medical issue, as various organs are involved, such as the liver, lung, heart, kidney, and skin, and its progression leads to organ failure and, especially in the liver, tumor development. The molecular mechanism of organ fibrosis has not yet been comprehensively clarified due to its complexity, and thus far, whether p53 is directly involved in its pathophysiology has not been addressed. Recently, 553 has been shown to accumulate in hepatocytes of several fibrotic liver diseases, such as

Authorship note: Takahiro Kodama and Tetsuo Takehara contributed equally to this work and share first authorship.

Conflict of interest: The authors have declared that no conflict of interest exists. Citation for this article: J Clin Invest. 2011;121(8):3343-3356. doi:10.1172/JCI44957. non-alcoholic steatohepatitis (NASH) $(8,9)$, viral hepatitis $(10,11)$, and primary biliary cirrhosis (PBC) (12). However, the precise role of p53 in liver fibrosis is unclear. To this end, in the present study, we generated mice with hepatocyte-specific deletion of $M d m 2$, a critical p53 inhibitor, which strictly maintains p53 at a low level by promoting $\mathrm{p} 53$ degradation via the ubiquitin/proteasome pathway (13). Studies in these mice revealed that hepatocyte p53 activation caused spontaneous liver fibrosis. In addition to increased hepatocyte apoptosis, these mice showed hepatocyte upregulation of connective tissue growth factor (CTGF), known to be the fibrogenic master switch in fibrotic liver diseases (14). In vitro study revealed that p53 induced CTGF synthesis in hepatocytes via microRNA (miRNA) regulation. Hepatocyte-specific knockdown of $p 53$ attenuated CTGF expression and liver fibrosis induced by an atherogenic (ATH) diet or TAA injection. In human liver samples, p53-regulated gene expression increased in the fibrotic liver in correlation with an increase in CTGF gene expression. These findings demonstrated for the first time to our knowledge that p53 is directly involved in fibrogenesis in association with the induction of profibrogenic gene expression, suggesting that hepatocyte p53 activation and subsequent CTGF upregulation could be therapeutic targets in fibrotic liver disease.

\section{Results}

Hepatocyte-specific Mdm2 deficiency causes endogenous p53 accumulation, leading to transactivation of $p 53$-regulated genes. To investigate the role of p53 in liver fibrosis, we first generated hepatocyte-specific Mdm2-knockout mice by crossing $M d m 2$ floxed mice $\left(M d m 2^{f / f l}\right)$ 
A

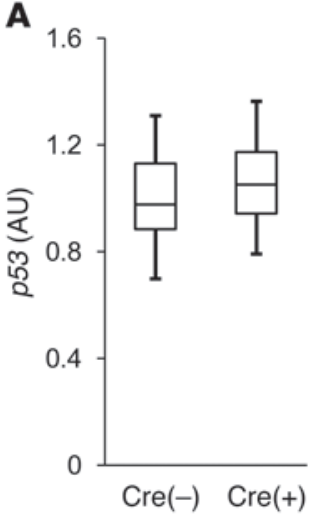

E

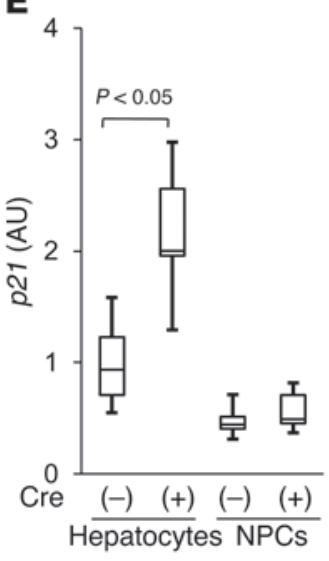

B
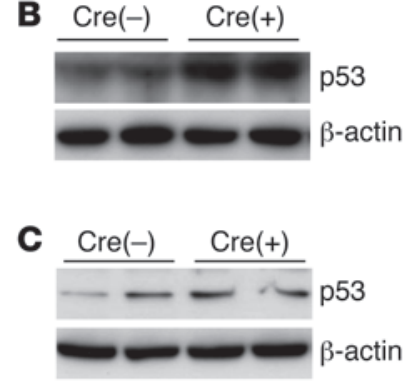

$(-) \quad(+) \quad(-) \quad(+)$ Nutlin-3a
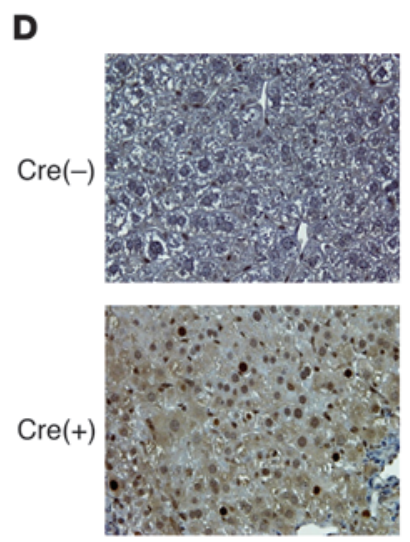

$\mathbf{F}$

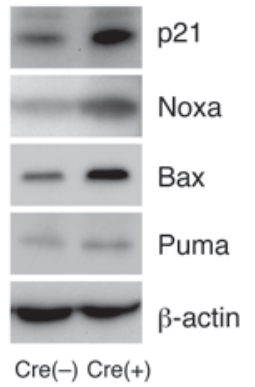

Figure 1

Hepatocyte-specific Mdm2-knockout mice show endogenous p53 accumulation, leading to transactivation of p53-regulated genes. (A-F) Mdm2 $2^{\text {fl/flallb-cre }}$ [Cre(+)] mice and $M d m 2^{f t / f t}[\mathrm{Cre}(-)]$ mice were analyzed at 6 weeks of age. (A) p53 mRNA levels in the liver tissue were determined by real-time RT-PCR; 7 mice per group. (B) Expression of p53 protein in liver tissue was assessed by Western blot analysis. (C) Expression of p53 protein in isolated hepatocytes upon treatment with $20 \mu \mathrm{M}$ nutlin-3a or vehicle was assessed by Western blot analysis. (D) Expression of p53 protein in the liver section was determined by immunohistochemical analysis. Original magnification, $\times 200$. (E) p21, Noxa, Bax, and Puma mRNA levels in isolated hepatocytes and NPCs were determined by real-time RT-PCR; 4 mice per group. Expression of p21, Noxa, Bax, and Puma proteins in liver tissue was assessed by Western blot analysis (F).

(15) and Alb-Cre transgenic mice (alb-cre) (16). After mating of $M d m 2^{f l /+}$ alb-cre mice with $M d m 2^{f l /+}$ mice, $M d m 2^{f l / f l}$ alb-cre mice were born at the expected Mendelian frequency and grew normally (Supplemental Figure 1; supplemental material available online with this article; doi:10.1172/JCI44957DS1). Next, we bred the $M d m 2^{f l / f l}$ alb-cre mice with the $M d m 2^{f / f l}$ mice and used $M d m 2^{f / f l}$ alb-cre mice as the knockout mice and $M d m 2^{f / f l}$ mice as control littermates in the subsequent experiments. We examined whether Mdm2 deficiency would cause p53 accumulation in the liver. Real-time RT-PCR study revealed that hepatic levels of p53 mRNA were not significantly different in the knockout mice and the control littermates (Figure 1A). Western blot analysis showed that hepatic p53 protein increased in the knockout mice compared with control littermates (Figure 1B). To determine whether an increase in p53 occurs in hepatocytes, we isolated hepatocytes from the liver by the collagenase-pronase perfusion procedure (17) and then examined their expression of $\mathrm{p} 53$ protein. Western blot analysis showed that the levels of hepatocyte p53 protein were higher in the knockout mice than in the control littermates (Figure 1C). These findings indicated that hepatocyte-specific Mdm2-knockout mice exhibited accumulation of $\mathrm{p} 53$ protein in their hepatocytes independent of the transcriptional upregulation of the $p 53$ gene. In addition, p53 expression increased in hepatocytes isolated from the control littermates, but not from the knockout mice, upon treatment with nutlin-3a, a small molecule $\mathrm{Mdm} 2$ inhibitor that blocks p53-Mdm2 interaction (ref. 18 and Figure 1C). This result demonstrated that lack of the Mdm2 function in hepatocytes of the knockout mice led to accumulation of endogenous p53 protein. Immunohistochemical examination of the liver sections revealed that p53 protein had accumulated in hepatocytes of the knockout mice, with some nuclear localization (Figure 1D), suggesting that p53 may become functionally active in hepatocytes of the knockout mice. This led us to investigate whether the p53 accumulation would lead to transactivation of p53-regulated genes p21, Noxa, Bax, and Puma. Real-time RT-PCR study revealed that, among these genes, the expression levels of $p 21$, Noxa, and Bax was significantly higher in hepatocytes of the knockout mice than the control littermates (Figure 1E). Western blot study demonstrated that protein levels of these p53-regulated genes increased in the knockout mice as well (Figure 1F). These results demonstrated that hepatocyte-specific Mdm2 deletion led to p53 accumulation and caused functional activation of p53 in hepatocytes. 
A

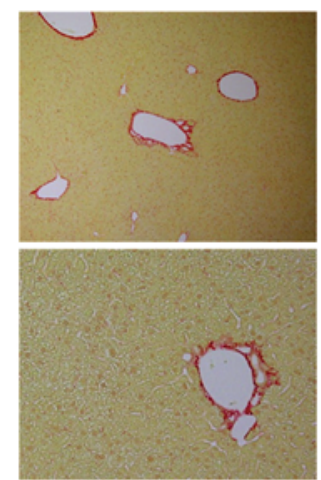

Cre (-)
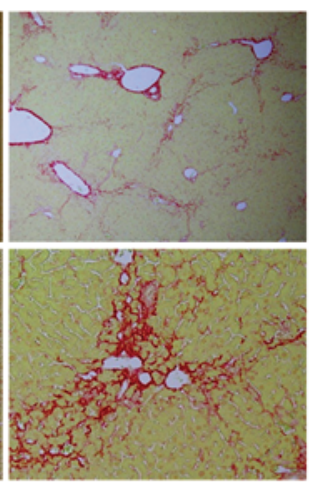

Cre $(+)$
D

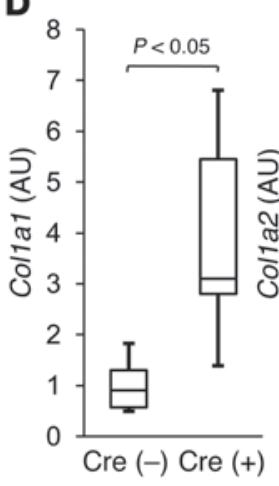

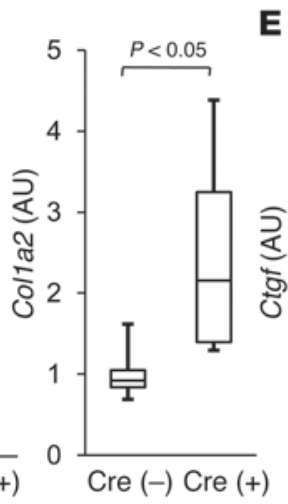
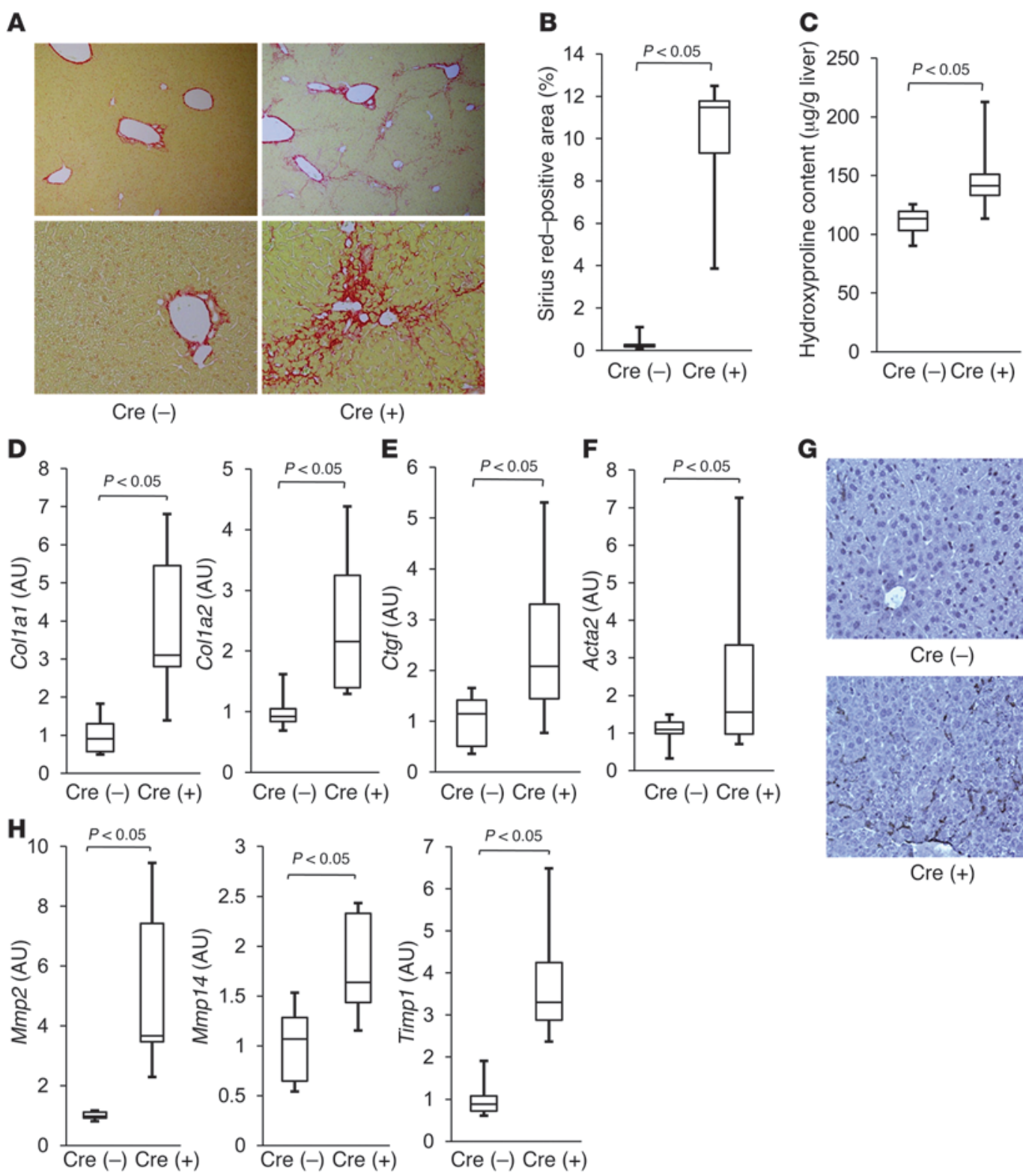

$\mathbf{F} \quad \mathbf{G}$
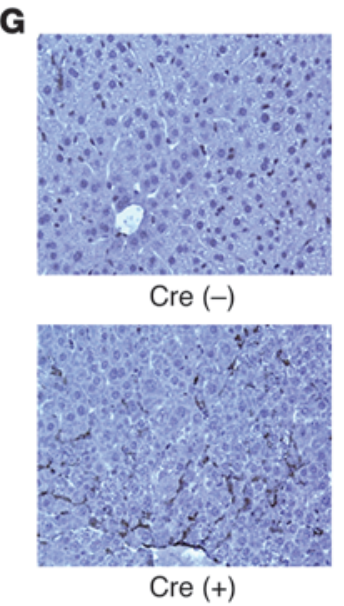

Figure 2

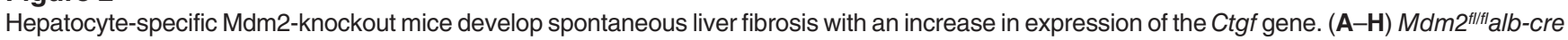
[Cre(+)] mice and $M d m 2^{t / f t}[$ [Cre(-)] mice were analyzed at 6 weeks of age; 6 mice per group. (A) Liver fibrosis was evaluated by picrosirius red staining of liver sections (original magnification, upper panels, $\times 100$; lower panels, $\times 200$ ). (B) Sirius red-positive area of liver sections. (C) Hepatic hydroxyproline content. Col1a1 and Col1a2 (D), Ctgf (E), and Acta2 (F) mRNA levels in the liver were determined by real time RT-PCR. (G) Expression of $\alpha$-SMA in the liver sections was analyzed by immunohistochemistry. Original magnification, $\times 200$. (H) Mmp2, Mmp14, and Timp1 mRNA levels in the liver were determined by real time RT-PCR.

Hepatocyte-specific Mdm2-knockout mice develop spontaneous liver fibrosis with an increase in Ctgfgene expression. We next examined the consequences of hepatocyte p53 activation in the liver of Mdm2knockout mice. To assess liver fibrosis, we evaluated hepatic collagen deposition by picrosirius red staining of liver tissues. At 6 weeks of age, pericellular and periportal bridging fibrosis was observed in liver of the knockout mice (Figure 2A), and it persisted even at a later time point (Supplemental Figure 2). Their collagen deposition significantly increased compared with that in the control littermates (Figure 2B). Hepatic hydroxyproline content, a biochemical marker of collagen accumulation (16), was also significantly higher in the knockout mice than in the wild-type mice (Figure 2C). We examined hepatic expression of the type I collagen genes Col1a1 and Col1a2 and found it to be significantly higher in the knockout mice than in the control littermates (Figure 2D). Among the major profibrogenic genes, real-time RT-PCR study revealed that hepatic expression of Ctgf was significantly higher in the knockout mice than in the control littermates (Figure 2E). Although Tgfb1 and Pdgfb gene expression was slightly higher in the knockout mice than in the control litter- 
A

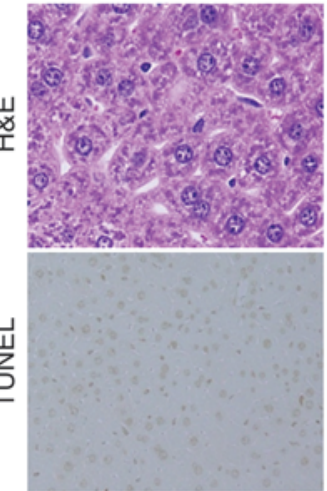

Cre(-)

D
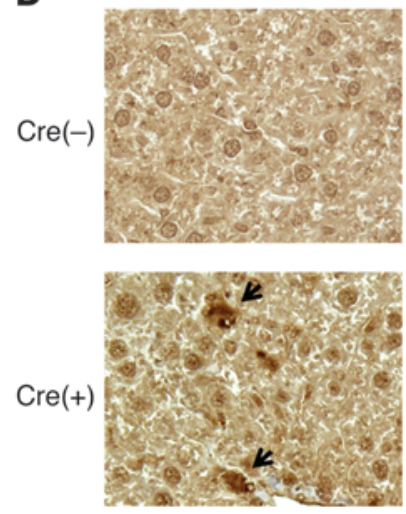

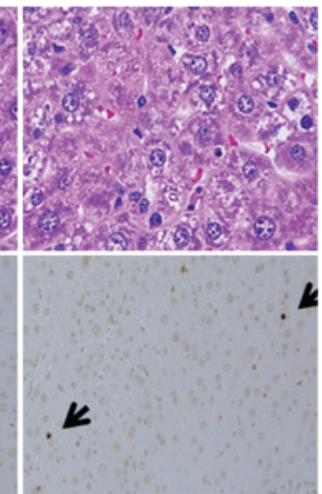

Cre(+)

E

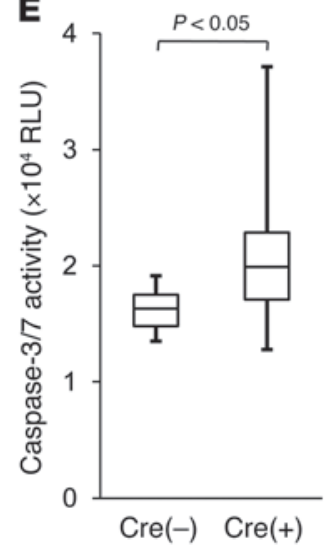

B

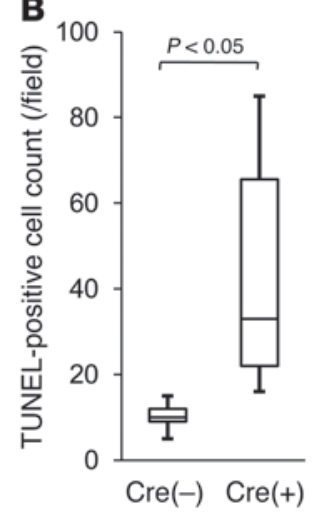

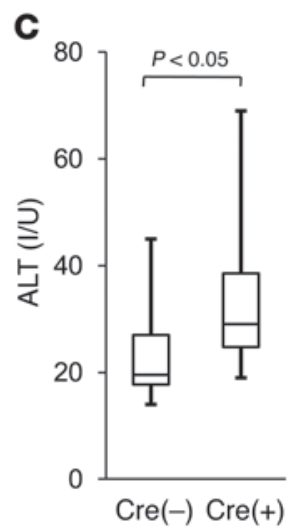

Figure 3

Hepatocyte-specific $M d m 2$ deletion induces modest hepatocyte apoptosis. (A-E) Mdm2 $2^{f / / f l}$ allb-cre $[\mathrm{Cre}(+)]$ mice and $M d m 2^{f / / f l}[\mathrm{Cre}(-)]$ mice were examined at 6-8 weeks of age; more than 6 mice per group. (A) Hepatocyte apoptosis was evaluated by H\&E staining and TUNEL staining of liver sections; black arrows indicate TUNEL-positive cells. Original magnification, upper panels, $\times 400$; lower panels, $\times 200$. (B) TUNEL-positive cell counts of liver sections. (C) Serum levels of ALT. (D) Expression of cleaved caspase-3 protein in the liver sections was assessed by immunohistochemistry; black arrows indicate cleaved caspase-3-positive cells. Original magnification, $\times 400$. (E) Serum caspase-3/7 activity. RLU, relative light units.

mates, the difference was not significant (Supplemental Figure 3). These findings indicated that hepatocyte-specific $M d m 2$ deletion led to spontaneous liver fibrosis with an increase in hepatic Ctgf gene expression. Activated hepatic stellate cells (HSCs), which express myogenic markers such as $\alpha$-SMA, are major collagen-producing cells in the injured liver (19). We thus examined whether activated HSCs were involved in the spontaneous fibrosis of the knockout mice. Real time RT-PCR demonstrated that hepatic expression of the $\alpha$-SMA gene Acta2 was significantly higher in the knockout mice than in control littermates (Figure $2 \mathrm{~F}$ ), and immunohistochemical study revealed that $\alpha$-SMA-positive cells increased in the liver of the knockout mice (Figure 2G), indicating that activated HSCs increased in the liver of the knockout mice. Liver fibrosis is known to be regulated by a fine balance between fibrogenesis and fibrinolysis, with activated HSCs playing a central role $(19,20)$. Real-time RT-PCR study showed that expression of fibrinolysis-related genes such as Mmp2, Mmp14, and Timp1, which are mainly produced from activated HSCs, also increased and was significantly higher in the knockout mice than in the control littermates (Figure $2 \mathrm{H}$ ). These findings suggested the involvement of activated HSCs in regulation of the fibrosis phenotype in liver of the knockout mice.
Hepatocyte-specific Mdm2 deletion induces modest apoptosis, but regenerative capacity remains normal. p53 activation is known to induce apoptosis, cell-cycle arrest, and senescence in a variety of tissues (1). We examined apoptotic phenotypes in liver of the knockout mice. H\&E staining of liver tissue revealed that a small number of hepatocytes with chromatin condensation and cytosolic shrinkage were scattered in the liver lobules of the knockout mice, with mild hepatic infiltration of inflammatory cells (Figure 3A). TUNEL staining of the liver tissue revealed an increase in TUNEL-positive cells in the knockout mice compared with the control littermates (Figure 3, A and B). Consistent with these histological observations, the levels of serum alanine aminotransferase (ALT), an indicator of liver injury, were slightly but significantly higher in the knockout mice than in the control littermates (Figure 3C). We also found that cleaved caspase-3, an active form of caspase-3, appeared in scattered hepatocytes of the knockout mice (Figure 3D), and that serum caspase-3/7 activity, which can be used as an indicator of hepatocyte apoptosis $(21,22)$, was significantly higher in the knockout mice than in the control littermates (Figure 3E). These findings indicated that hepatocyte-specific deletion of $M d m 2$ led to a modest increase in spontaneous hepatocyte apoptosis. Next, we investigated the regenerative status of the liver upon $70 \%$ partial 
A

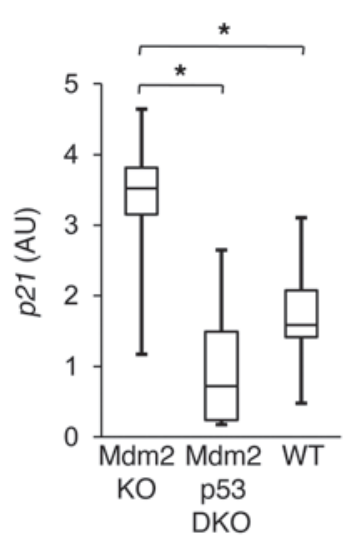

B

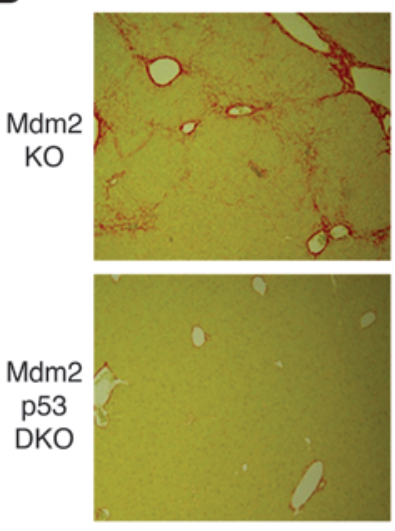

C

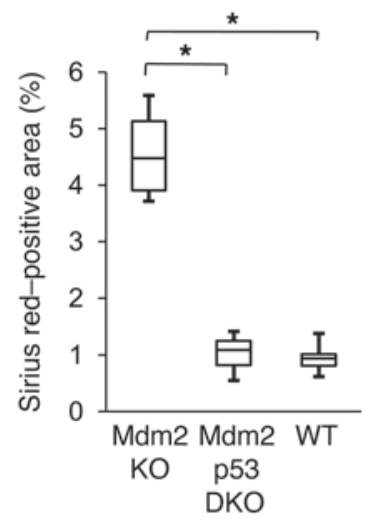

D

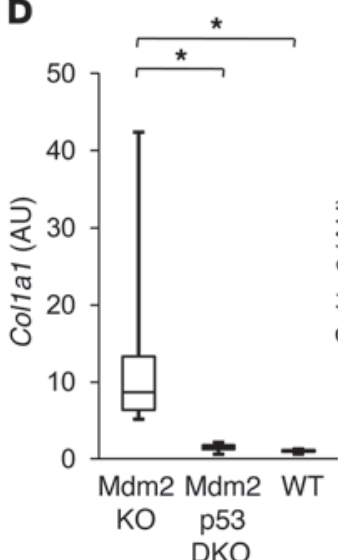

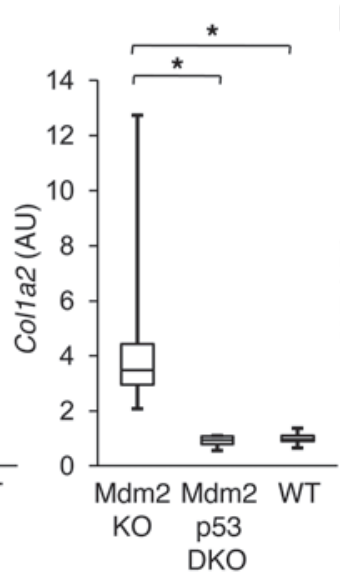

E

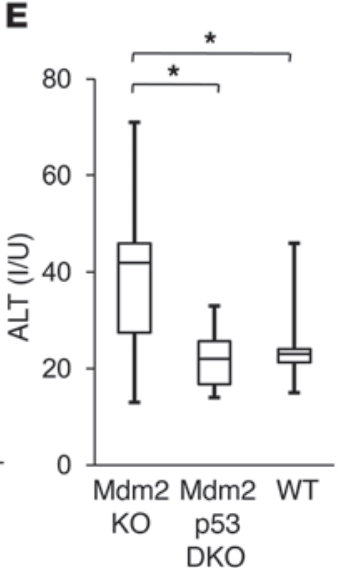

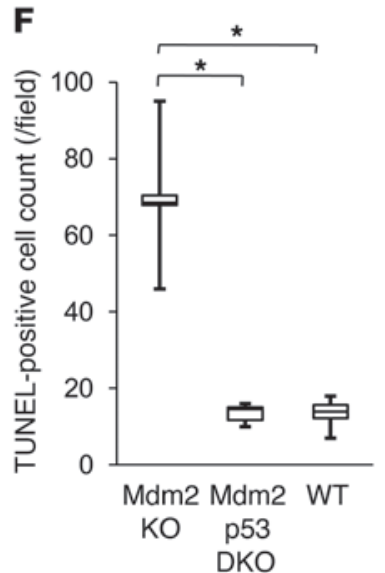

Figure 4

Spontaneous liver fibrosis in hepatocyte-specific Mdm2-knockout mice is completely abolished in a hepatocyte-specific p53-knockout back-

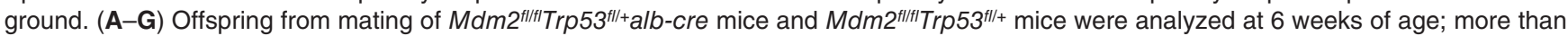

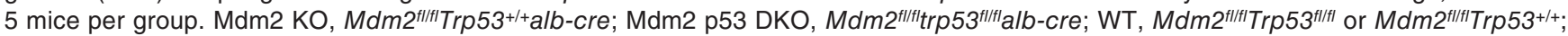
${ }^{*} P<0.05$. (A) $p 21$ mRNA levels in the liver were determined by real-time RT-PCR. (B) Liver fibrosis was evaluated by picrosirius red staining of liver sections. Original magnification, $\times 100$. (C) Sirius red-positive area of liver sections. (D) Col1a1 and Col1a2 mRNA levels in the liver were determined by real-time RT-PCR. (E) Serum levels of ALT. (F) Hepatocyte apoptosis was evaluated by TUNEL staining of liver sections. (G) Ctgf mRNA levels in the liver were determined by real-time RT-PCR. ${ }^{*} P<0.05$.

hepatectomy, a well-established model of liver regeneration (23), by hepatic BrdU uptake and H\&E staining of the liver tissue. Upon partial hepatectomy, compensatory liver regeneration occurred in both groups compared with the sham operation group, and the difference between them was not significant (Supplemental Figure 4, $\mathrm{A}$ and B). Even at a later time point, upon hepatectomy, liver volume steadily recovered in both groups and did not differ between them (Supplemental Figure 4C). These results indicated that hepatocyte-specific Mdm2 deficiency did not affect the regenerative capacity of the liver of the knockout mice. Senescence-associated $\beta$-galactosidase staining of the liver sections was also performed and showed that senescent hepatocytes were not obvious in both groups at 6 weeks of age (Supplemental Figure 5).

Spontaneous liver fibrosis in hepatocyte-specific Mdm2-knockout mice is abolished in a hepatocyte-specific p53-knockout background. To investigate whether p53 activation in hepatocytes is responsible for the phenotypes observed in the Mdm2-knockout mice, we generated hepatocyte-specific Mdm2- and p53-double-knockout mice by crossing hepatocyte-specific Mdm2-knockout mice ( $M d m 2^{f / f l}$ alb-cre $)$ and p53 floxed mice $\left(\operatorname{Trp} 53^{f l f l f}\right)$. After mating of $M d m 2^{f l / f l} \operatorname{Tr} p 53^{f l /+}$ alb-cre mice with $M d m 2^{f / f l} \operatorname{Trp} 53^{f / /+}$ mice, hepatocyte-specific Mdm2- and p53-double-knockout mice ( $M d m 2^{f / f l} \operatorname{Trp} 53^{f / f l}$ alb-cre $)$ were born at the expected Mendelian frequency and grew normally (Supplemental Figure 6). Levels of the p21 gene, as the p53-regulated gene, were significantly lower in the hepatocyte-specific Mdm2- and p53-double-knockout mice than in the hepatocyte-specific Mdm2-knockout littermates $\left(M d m 2^{f / f l} \operatorname{Trp} 53^{+/+}\right.$alb-cre $)$and were not significantly different from those in wild-type littermates $\left(M d m 2^{f / / f l} \operatorname{Trp} 53^{+/+}\right.$or $M d m 2^{f / f l} \operatorname{Tr} p 53^{f / f l}$ ) (Figure 4A). Picrosirius red staining of the liver tissue demonstrated that spontaneous liver fibrosis was completely abolished in the double-knockout mice (Figure 4B) and collagen deposition was significantly lower in the double-knockout mice than in the Mdm2knockout littermates (Figure 4C). Type I collagen gene expression also significantly decreased in the double-knockout mice compared with the single-knockout mice and was not different from that in wild-type littermates when assessed by real-time RT-PCR (Figure 4D). These findings clearly demonstrated that the spontaneous liver fibrosis in the Mdm2-knockout mice was completely dependent on p53, 

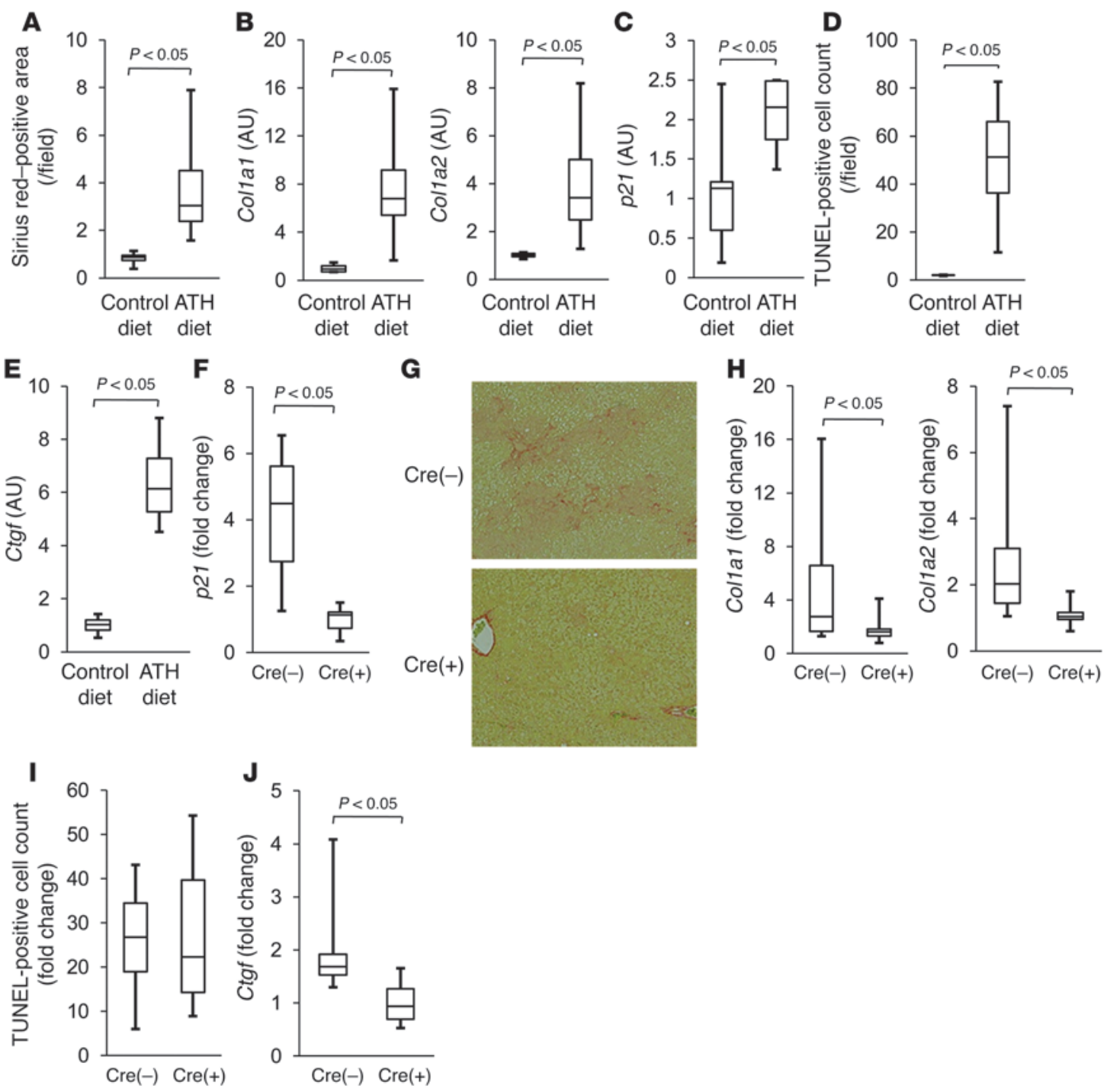

\section{Figure 5}

Hepatocyte-specific p53-knockout mice show alleviated liver fibrosis induced by ATH diet with suppression of the CTGF increase. (A-E) C57BL6/J mice were fed ATH diet or control diet for 4 weeks and then examined; 4 mice per group. (A) Liver fibrosis was evaluated by picrosirius red staining of liver sections. (B) Col1a1 and Col1a2 mRNA levels in the liver were determined by real-time RT-PCR. (C) p21 mRNA levels in the liver were determined by real-time RT-PCR. (D) Hepatocyte apoptosis was evaluated by TUNEL staining of liver sections. (E) Ctgf mRNA levels in the liver were determined by real-time RT-PCR. $(\mathbf{F}-\mathbf{J}) \operatorname{Trp53^{f//fI}}[(\mathrm{Cre}(-)]$ mice and Trp53 fl/flallb-cre [Cre(+)] mice were fed ATH diet or control diet for 4 weeks and then examined; more than 6 mice per group; data are presented as fold change in the ATH diet group compared with the control diet group. (F) p21 mRNA levels in the liver were determined by real-time RT-PCR. (G) Liver fibrosis was evaluated by picrosirius red staining of the liver sections. Original magnification, $\times 100$. (H) Col1a1 and Col1a2 mRNA levels in the liver were determined by real-time RT-PCR. (I) Hepatocyte apoptosis was evaluated by TUNEL staining of liver sections. (J) Ctgf mRNA levels in the liver were determined by real-time RT-PCR.

indicating that endogenous p53 activation in hepatocytes causes spontaneous liver fibrosis. ALT levels were normalized in the doubleknockout mice, with a significant decrease in TUNEL-positive cells in the liver (Figure 4, E and F). Ctgf gene expression was also significantly lower in the double-knockout mice than in the single-knockout mice and was not different from that in wild-type littermates (Figure 4G). These results indicated that hepatocyte $\mathrm{p} 53$ activation induced hepatocyte apoptosis and CTGF upregulation in the liver.

Hepatocyte-specific p53-knockout mice show alleviated liver fibrosis induced by ATH diet with suppression of CTGF increase. To investigate the involvement of p53 in liver fibrosis, we examined p53 activation in liver of wild-type mice fed an ATH diet, an experimental model of murine liver fibrosis $(24,25)$. After 4 weeks of ATH diet feeding, wild-type mice developed liver fibrosis as assessed by hepatic collagen deposition of picrosirius red staining, with upregulation of Col1a1 and Col1a2 gene expression (Figure 5, A and B). Regarding the p53-regulated genes, real-time RT-PCR analysis revealed that, in liver of the ATH diet-fed mice, $p 21$ gene expression levels rose and were significantly higher than those in liver of control diet-fed mice (Figure 5C). This finding suggested that p53 activation had occurred in the liver fibrosis induced by the ATH diet. TUNEL staining of the liver sections showed that hepatocyte 

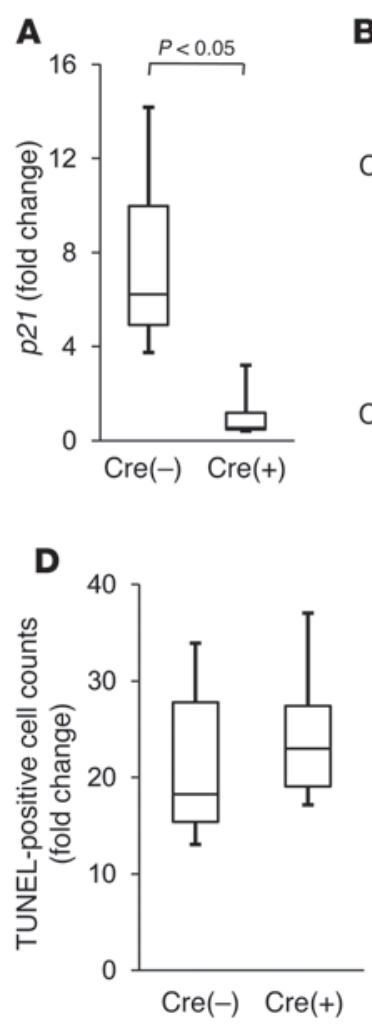

$\mathbf{B}$

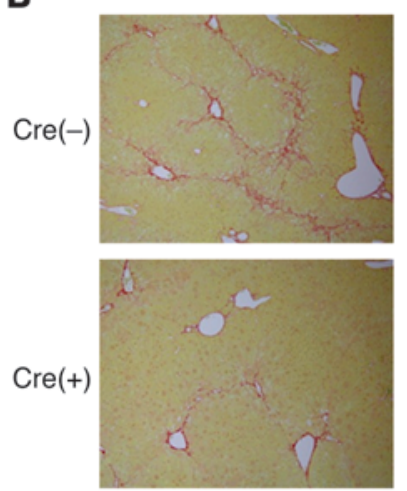

E

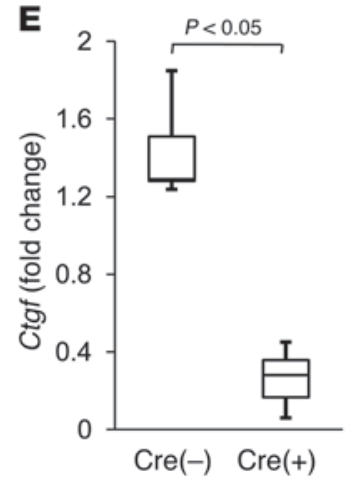

C
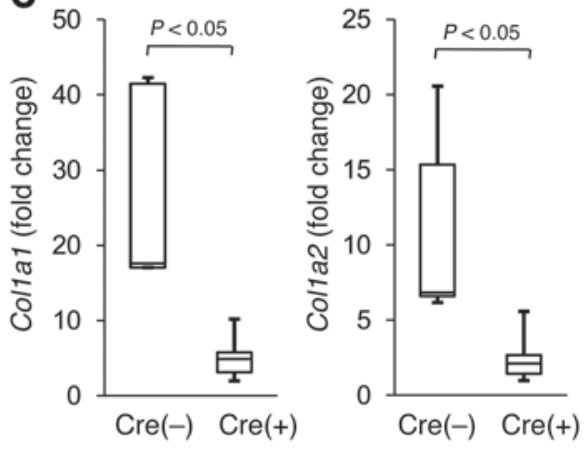

\section{Figure 6}

Hepatocyte-specific p53-knockout mice show alleviated liver fibrosis induced by TAA administration, with suppression of the CTGF increase. (A-E) Trp53 ${ }^{f / / f l}[\mathrm{Cre}(-)]$ mice and Trp53 fl/flallb-cre [Cre(+)] mice were given intraperitoneal injection of $200 \mathrm{mg} / \mathrm{kg}$ TAA 3 times per week for 6 weeks and then analyzed; 6 mice per group; data are presented as fold change in the TAA-treated group compared with the nontreated group. (A) p21 mRNA levels in the liver were determined by real-time RT-PCR. Original magnification, $\times 100$. (B) Liver fibrosis was evaluated by picrosirius red staining of the liver sections. (C) Col1a1 and Col1a2 mRNA levels in the liver were determined by real-time RT-PCR. (D) Hepatocyte apoptosis was evaluated by TUNEL staining of liver sections. (E) Ctgf mRNA levels in the liver were determined by real-time RT-PCR.

apoptosis significantly increased in ATH diet-fed mice compared with the control diet-fed mice (Figure 5D). Moreover, with this ATH diet, Ctgf gene expression significantly increased in the liver (Figure 5E). To investigate whether p53 activation was involved in the progression of liver fibrosis provoked by the ATH diet, the hepatocyte-specific p53-knockout mice (Trp5 $3^{f / f l}$ alb-cre) and the control littermates $\left(\operatorname{Trp} 53^{f / f l}\right)$ were fed the ATH diet or control diet, and then liver fibrosis was examined. After 4 weeks of feeding on the ATH diet, the $p 21$ gene was upregulated in the control littermates but not in the knockout mice, thus confirming p53 activation in hepatocytes in this fibrosis model (Figure 5F). Picrosirius red staining of the liver tissues revealed that liver fibrosis was alleviated in the knockout mice compared with the control littermates (Figure 5G). Real-time RT-PCR study demonstrated that the ATH diet-induced increase in Col1a1 and Col1a2 gene expression was significantly attenuated in the knockout mice compared with control littermates (Figure $5 \mathrm{H}$ ). These results indicated that inhibition of p53 activation in hepatocytes alleviated the liver fibrosis caused by the ATH diet. With this ATH diet, hepatocyte apoptosis increased similarly in both groups compared with the control diet, and there was no significant difference between them in the increase, when assessed by TUNEL staining of the liver tissue (Figure 5I). This finding suggested that p53-dependent hepatocyte apoptosis was not much involved in this model. On the other hand, while the ATH diet upregulated Ctgf gene expression in the control litter- mates, this did not occur in the knockout mice (Figure 5J), suggesting that p53-mediated CTGF upregulation may be involved in the progression of liver fibrosis caused by the ATH diet.

Hepatocyte-specific p53-knockout mice show alleviated liver fibrosis induced by thioacetamide administration, with suppression of the increase in CTGF. To further investigate the involvement of p53 in another well-established model of liver fibrosis, we used repetitive intraperitoneal injection of thioacetamide (TAA) (26) to examine the hepatocyte-specific p53-knockout mice and control littermates. Upon 6 weeks of TAA administration, $p 21$ gene expression increased in the control littermates but not in the knockout mice, and there was a significant difference between them in its upregulation (Figure 6A). These findings suggested that p53 activation occurred in this fibrosis model as well. Picrosirius red staining of the liver sections revealed that liver fibrosis was alleviated in the knockout mice compared with control littermates (Figure 6B). Real-time RT-PCR study demonstrated that TAA-induced increases in Col1a1 and Col1a2 gene expression were significantly attenuated in the knockout mice compared with control littermates (Figure 6C). These results indicated that inhibition of p53 activation in hepatocytes alleviated TAA-induced liver fibrosis. TAA treatment increased hepatocyte apoptosis in both groups to a similar extent, as assessed by TUNEL staining of the liver tissue (Figure 6D). On the other hand, upon TAA treatment, there was a significant difference between them in the CTGF increase 

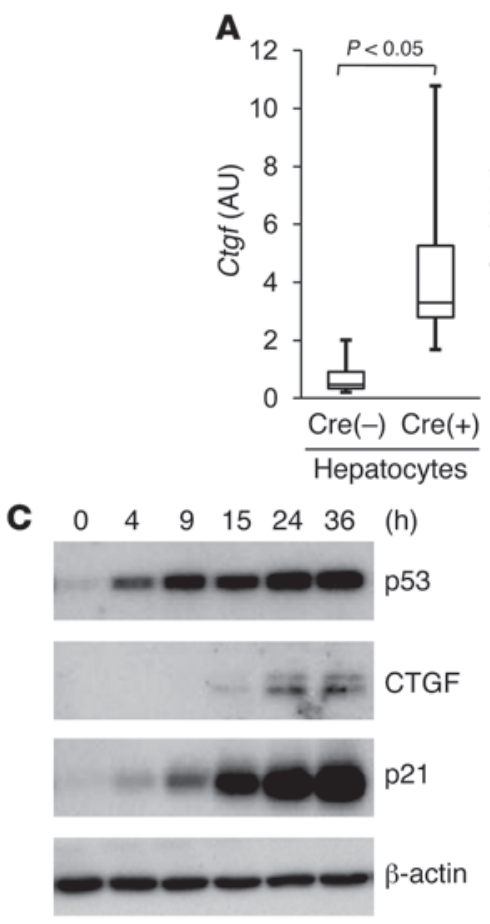

G

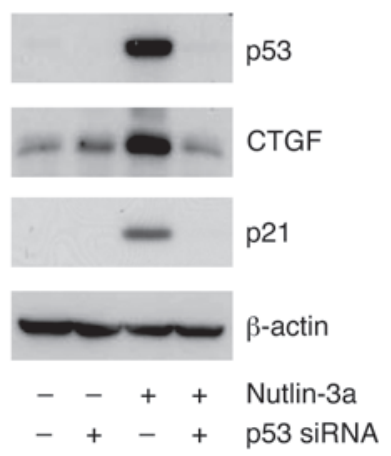

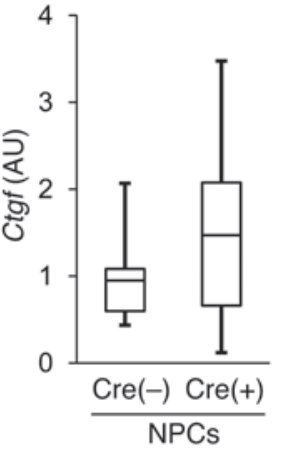

D
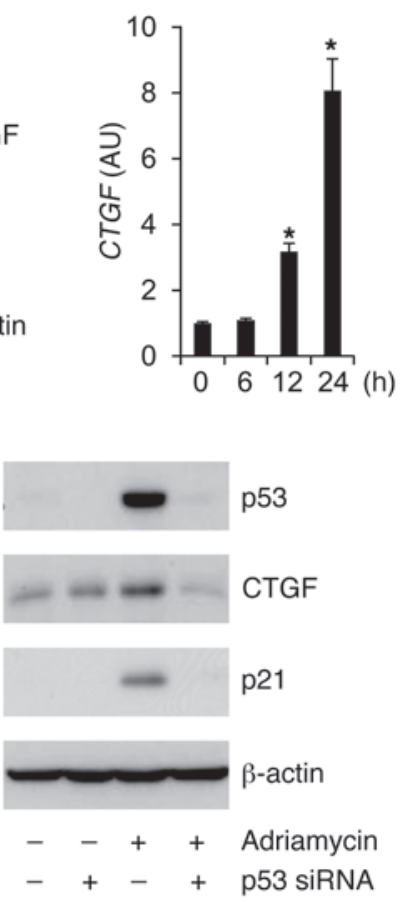

B

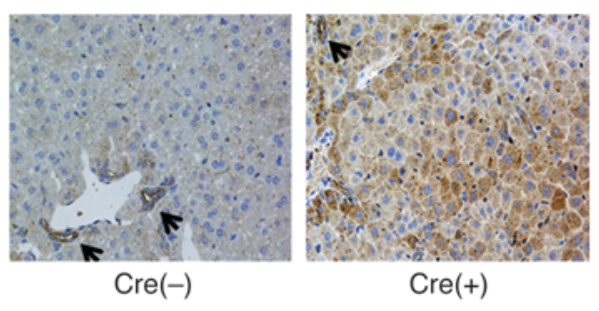

E

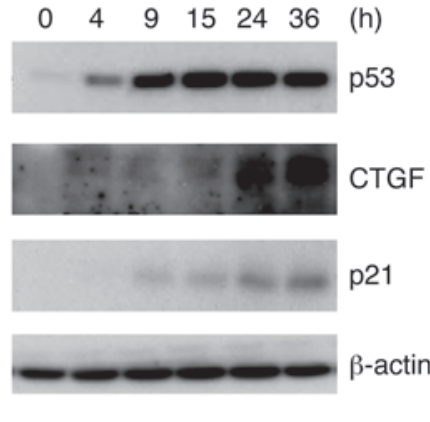

$\mathbf{F}$

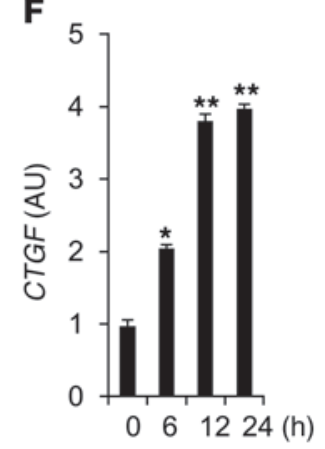

H $\quad$ anC siRnA
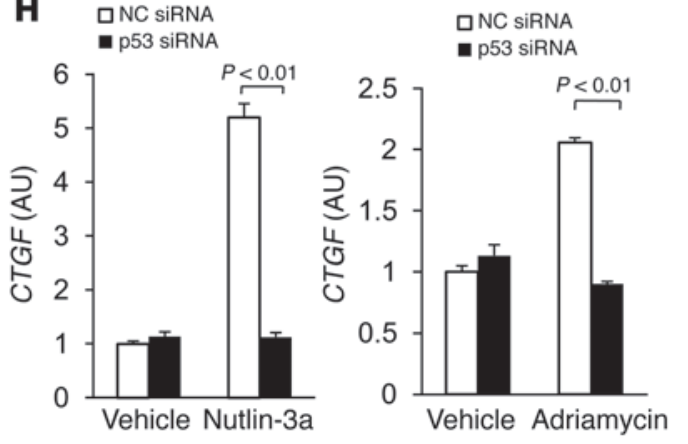

Figure 7

p53 regulates CTGF synthesis in hepatocytes. (A) Hepatocytes and NPCs were isolated from Mdm2 $2^{f / f f l}[\mathrm{Cre}(-)]$ mice and $M d m 2^{f / / f l a l b-c r e ~[C r e(+)] ~}$ mice by collagenase-pronase perfusion of the liver. Ctgf mRNA levels in the isolated hepatocytes (left panel) and NPCs (right panel) were determined by real-time RT-PCR; 4-6 mice per group. (B) Expression of CTGF protein in liver sections was assessed by immunohistochemistry; black arrows indicate cholangiocytes. Original magnification, $\times 200$. (C and D) HepG2 cells $\left(1.0 \times 10^{5}\right)$ were treated with nutlin-3a $(20 \mu \mathrm{M})$ or vehicle for the indicated time courses. (C) Western blot analysis of p53, CTGF, and p21 proteins. (D) Real-time RT-PCR analysis of CTGF mRNA expression; $n=3$ /group; ${ }^{*} P<0.01$ versus the other 3 groups. $(\mathbf{E}$ and $\mathbf{F})$ HepG2 cells $\left(1.0 \times 10^{5}\right)$ were treated with Adriamycin $(1 \mu \mathrm{M})$ or vehicle for indicated time courses. (E) Western blot analysis of p53, CTGF, and p21 proteins. (F) Real-time RT-PCR analysis of CTGF expression; $n=3 /$ group; ${ }^{*} P<0.01$ versus the other 3 groups, ${ }^{* \star} P<0.01$ versus 0 - and 6 -hour groups. ( $\mathbf{G}$ and $\mathbf{H}$ ) HepG2 cells were transfected with $p 53$ siRNA or control siRNA for 3 days and then cultured for 24 hours with nutlin-3a $(20 \mu \mathrm{M})$, Adriamycin $(1 \mu \mathrm{M})$, or vehicle. (G) Western blot analysis of p53, CTGF and p21 proteins. (H) Real-time RT-PCR analysis of CTGF mRNA expression; $n=3 /$ group, statistical analyses were performed by the paired $t$ test.

(Figure 6E). These findings suggested that p53-mediated CTGF upregulation may be also involved in the progression of liver fibrosis provoked by TAA treatment.

p53 regulates CTGF synthesis in hepatocytes. We tried to identify the cells in which CTGF synthesis increased in the liver of hepatocyte-specific Mdm2-knockout mice. Ctgf gene expression in the hepatocytes of the knockout mice was significantly higher than in the control littermates (Figure 7A), while it did not significantly differ between them in the non-parenchymal cells (NPCs) (Figure 7A). Immunohistochemical examinations in the liver sections also revealed that CTGF was expressed in hepatocytes of the knockout mice, but not in those of control littermates (Figure 7B). On the other hand, CTGF was expressed in cholangiocytes of both groups of mice, but its levels were not much different between them. These findings suggested that p53 activation induced CTGF synthesis in murine hepatocytes. Next, to investigate the involvement of p53 in CTGF regulation in human hepatocytes, we performed an in vitro study using HepG2 cells, which are known to preserve wild-type p53 function (27). Administration of nutlin-3a into HepG2 cells led to time-dependent increases in p53 and p53-regulated gene 

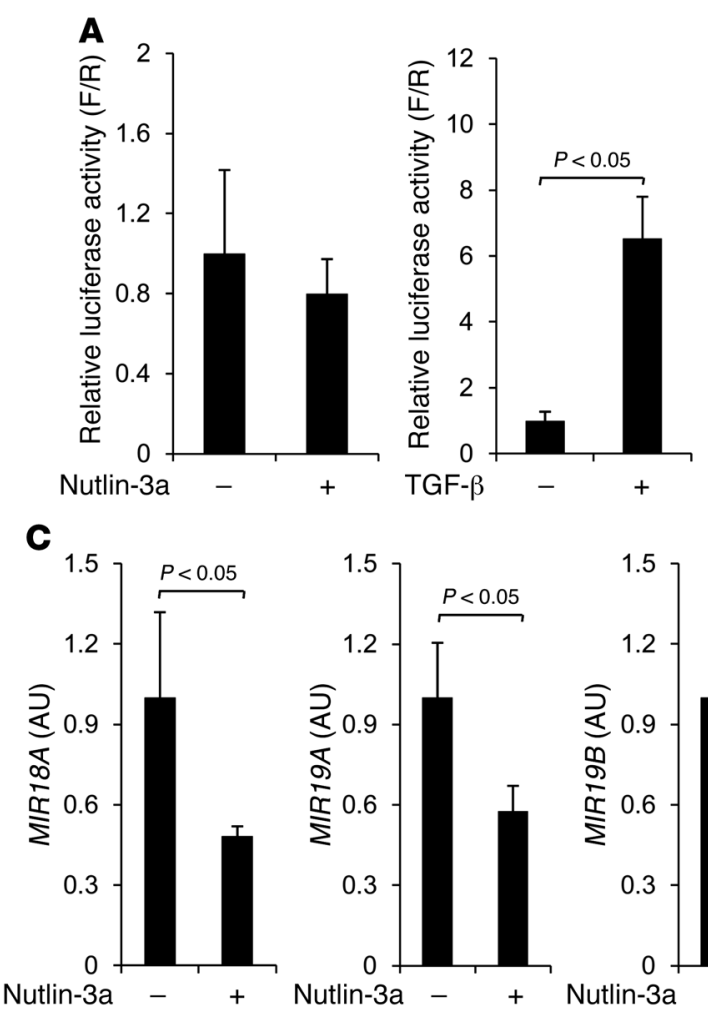

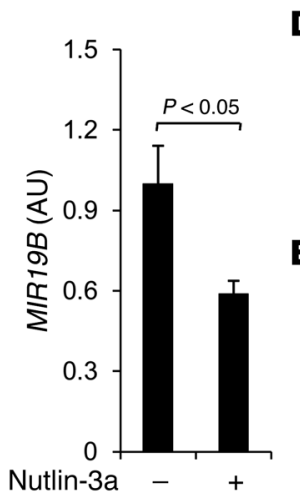

B
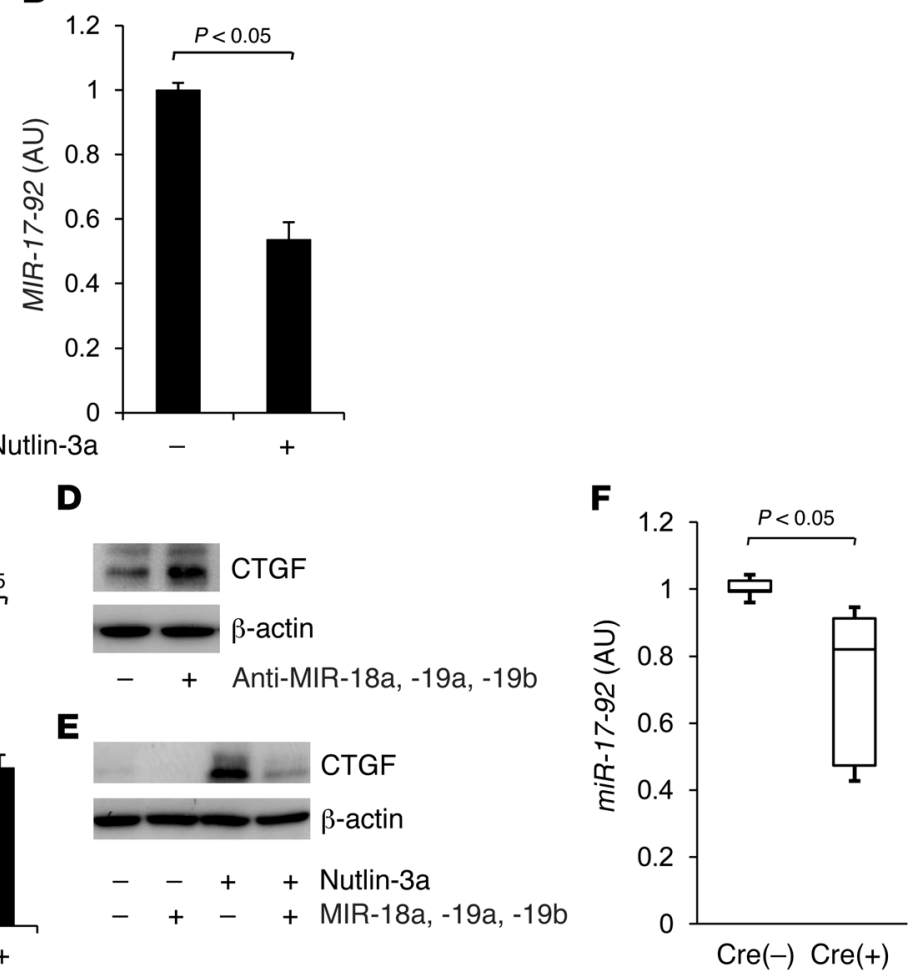

Figure 8

p53 activation upregulates CTGF synthesis via repression of the miR-17-92 cluster gene. (A) HepG2 cells $\left(1.0 \times 10^{5}\right)$ were cotransfected with pTS-589 and pRL-TK for 48 hours and treated with nutlin-3a $(20 \mu \mathrm{M})$ or recombinant TGF- $\beta(10 \mathrm{ng} / \mathrm{ml})$ for 24 hours. Firefly luciferase and Renilla luciferase activity was measured and is presented as relative luminescence values for firefly luciferase versus Renilla luciferase $(F / R) . ~ n=4 /$ group. (B and C) HepG2 cells $\left(1.0 \times 10^{5}\right)$ were treated with nutlin-3a $(20 \mu \mathrm{M})$ or vehicle for 24 hours. (C) Real-time RT-PCR analysis of miR-17-92 mRNA (B), MIR18A, MIR19A, and MIR19B miRNA expression; $n=3 /$ group. Statistical analyses were performed by the paired $t$ test (A-C). (D) HepG2 cells were transfected with a mixture of antisense of MIR18A, MIR19A, and MIR19B at $100 \mathrm{nM}$ each or negative control at $300 \mathrm{nM}$ for 2 days. Expression of CTGF protein was assessed by Western blotting. (E) HepG2 cells were transfected with a mixture of precursor of MIR18A, MIR19A, and MIR19B at $10 \mathrm{nM}$ each or negative control at $30 \mathrm{nM}$ for 2 days and cultured with nutlin-3a (20 $\mu \mathrm{M})$ or vehicle for 24 hours. Expression of CTGF protein was assessed by Western blotting. (F) Expression of miR-17-92 mRNA in isolated hepatocytes was assessed by

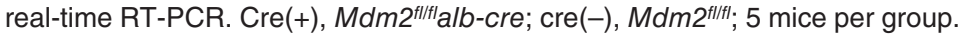

products, represented by $\mathrm{p} 21$ (Figure 7C), indicating that nutlin-3a could activate p53 in these cells. Upon nutlin-3a treatment, CTGF gene expression increased in a time-dependent manner in HepG2 cells (Figure 7D), while TGFB1 and PDGFB gene expression did not (Supplemental Figure 7). We also observed that CTGF protein levels gradually increased upon nutlin-3a treatment (Figure 7C). Adriamycin, a DNA-alkylating agent, could also activate p53, leading to upregulation of CTGF mRNA and protein levels in HepG2 cells in a time-dependent manner (Figure 7, E and F). Administration of p53 siRNA into HepG2 cells efficiently reduced p53 expression, which was demonstrated by the mRNA levels (Supplemental Figure 8) and protein levels (Figure 7G), and inhibited upregulation of $\mathrm{p} 21$ protein upon treatment with nutlin-3a and Adriamycin (Figure 7G). Under this condition, p53 knockdown completely abolished the increase in CTGF that had resulted from administration of these drugs (Figure $7, \mathrm{G}$ and $\mathrm{H}$ ). These results clearly demonstrated that the increase in CTGF synthesis by nutlin-3a or Adriamycin was completely dependent on p53 in HepG2 cells, indicating that $\mathrm{p} 53$ positively regulates CTGF synthesis in human hepatocytes. To directly demonstrate the effect of p53 on CTGF expression in vivo, we injected a p53 expression plasmid,
ORF9-mp53, or its control plasmid into BALB/c mice via the tail vein by a hydrodynamic injection procedure (28) and examined Ctof gene expression 2 days later. Although hydrodynamic injection of the p53 expression plasmid only led to nuclear expression of p53 in hepatocytes at a rate of approximately 5\% (Supplemental Figure 9A), it significantly increased Ctgf gene expression in the liver compared with the control hydrodynamic injection (Supplemental Figure 9B). These results also demonstrated the existence of the $\mathrm{p} 53$ /CTGF pathway in vivo.

p53 activation upregulates CTGF synthesis via repression of the miR-17-92 cluster gene. Next, we tried to elucidate the molecular mechanism underlying CTGF regulation by p53 in HepG2 cells. To examine whether p53 transcriptionally upregulates CTGF gene expression, we introduced plasmid pTS-589 - which contains the CTGF promoter from 802 base pairs upstream of the transcript start site to 22 base pairs downstream of the coding sequence linked to the upstream of a firefly luciferase reporter gene (29) - into HepG2 cells. Then, we evaluated the transcription activity of the CTGF promoter upon treatment with nutlin-3a or recombinant TGF- $\beta$, which is known to transcriptionally upregulate CTGF (29). Whereas luciferase activity increased upon TGF- $\beta$ treatment, it did not 
A

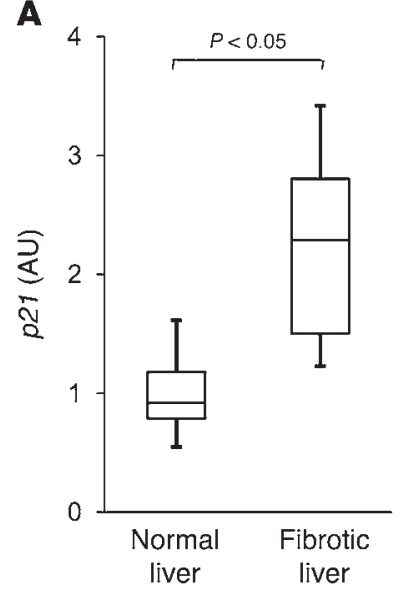

B

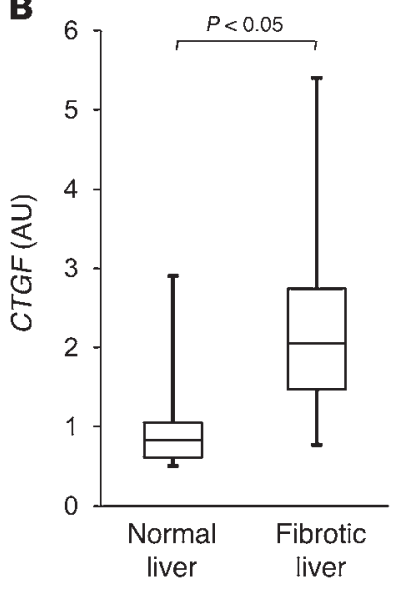

C

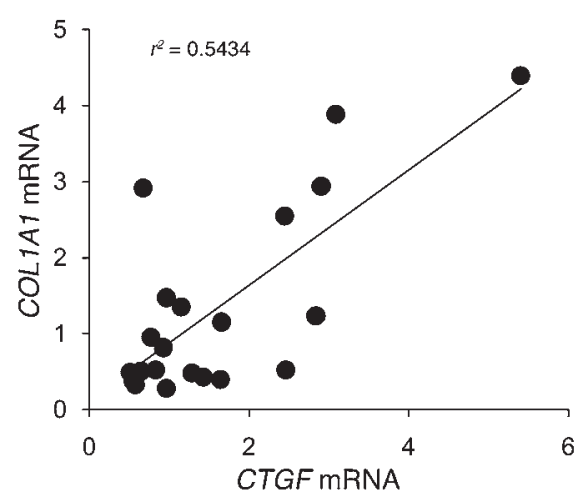

D

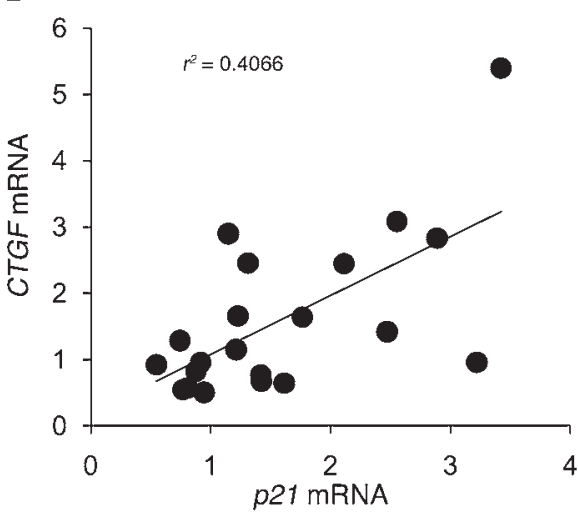

E

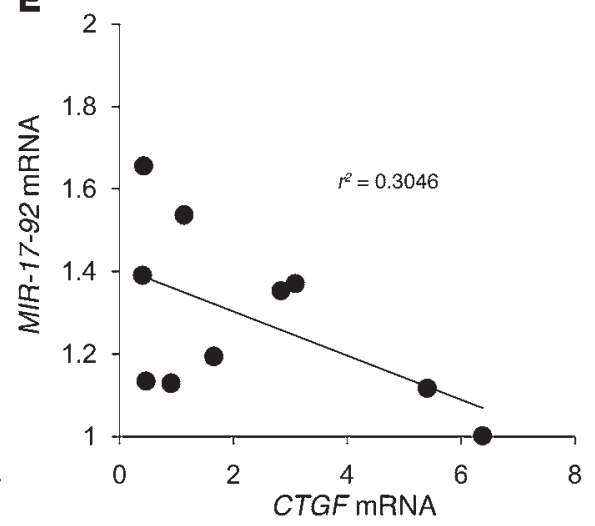

Figure 9

p53-regulated gene expression increases in fibrotic human liver and is correlated with an increase in CTGF gene expression. (A and B) A total of 21 non-tumorous human liver samples were subdivided into two groups histologically defined as normal liver and fibrotic liver. $p 21$ (A) and CTGF (B) mRNA levels in the liver were determined by real-time RT-PCR; $n=11$ (normal liver group) and $n=10$ (fibrotic liver group). (C and D) COL1A1, $C T G F$, and p21 mRNA levels in the liver of 21 non-tumorous human liver samples were determined by real-time RT-PCR and plotted to analyze the correlation between COL1A1 and CTGF $(P<0.01)(\mathbf{C})$ or between CTGF and $p 21(P<0.01)(\mathrm{D})$. (E) miR-17-92 and CTGF mRNA levels in the liver of 10 human fibrotic liver samples were determined by real-time RT-PCR and plotted to analyze the correlation between them $(P<0.01)$.

change upon nutlin-3a treatment in HepG2 cells (Figure 8A), suggesting that the post-transcriptional regulation may be involved in the p53-induced CTGF upregulation. Recently, epigenetic regulation of the CTGF gene has been demonstrated (30-32), and the miR-17-92 cluster gene, in particular, has been reported to repress CTGF synthesis in murine colonocytes and human glioblastoma cells $(31,32)$. Upon nutlin-3a treatment, expression of the miR-17-92 cluster gene decreased in HepG2 cells (Figure 8B), indicating that p53 activation reduced MIR-17-92 gene expression in HepG2 cells. The miR-17-92 cluster comprises 7 miRNAs that are transcribed as a single polycistronic unit $(31,33)$, and in silico analysis revealed that, among these miRNAs, MIR18A, MIR19A, and MIR19B can be predicted to target CTGF. Real-time RT-PCR study revealed that the gene expression of these 3 miRNAs also decreased upon nutlin-3a treatment in HepG2 cells (Figure 8C). Introduction of the antisense of these 3 miRNAs increased CTGF synthesis in HepG2 cells (Figure 8D), indicating that they suppressed CTGF synthesis in HepG 2 cells. To investigate the causal involvement of downregulation of the miRNAs in p53-induced CTGF upregulation, we administered precursors of the miRNAs to HepG2 cells, and CTGF synthesis was examined upon nutlin-3a treatment. In contrast to negative control miRNA, administration of these miRNAs inhibited the upregulation of CTGF upon nutlin-3a treatment (Figure 8E), suggesting that a decrease in miR-18a, miR-19a, and miR-19b in the miR-17-92 cluster was involved in the mechanism of CTGF upregulation by $\mathrm{p} 53$. To investigate the involvement of the miR-17-92 cluster in CTGF upregulation in hepatocytes of Mdm2-knockout mice, we examined hepatocyte gene expression of the miR-17-92 cluster and found it to be significantly lower in the knockout mice than in the control littermates (Figure $8 \mathrm{~F}$ ), suggesting that the miR-17-92 cluster may be involved in p53-mediated CTGF upregulation in hepatocytes of the knockout mice.

p53-regulated gene expression increases in the human fibrotic liver and is correlated with an increase in Ctgf gene expression. Finally, to investigate the relationship between p53 activation and human liver fibrosis, we examined the expression of $\mathrm{p} 53$-regulated genes and fibrosis-related genes in human liver samples. $p 21$ gene expression increased in the fibrotic liver and was significantly higher than in the normal liver (Figure 9A). We observed that NOXA gene expression in the fibrotic liver was also significantly higher than in the normal liver (Supplemental Figure 10). These findings suggested that p53 may be transcriptionally active in the fibrotic 
liver. Regarding fibrosis-related genes, CTGF gene expression was significantly higher in the fibrotic liver than in the normal liver (Figure 9B). The increase in CTGF gene expression paralleled the increase in COL1A1 gene expression, with a significant correlation between them (Figure 9C). These results suggested that CTGF may be involved in the progression of human liver fibrosis. There was a significant correlation between the gene expression of $p 21$ and CTGF (Figure 9D), suggesting that p53 activation might be involved in the upregulation of CTGF and the progression of liver fibrosis in humans. We also found that there was a significant negative correlation between the gene expression of CTGF and the $m i R-17-92$ cluster in the fibrotic liver (Figure 9E), suggesting the involvement of the miR-17-92 cluster in the p53/CTGF pathway in the human fibrotic liver.

\section{Discussion}

In the present study, to investigate the role of p53 in liver fibrosis, we generated hepatocyte-specific Mdm2-knockout mice and found a direct link between p53 activation in parenchymal cells and organ fibrosis. In unstressed cells, expression of p53 is tightly regulated and maintained at a low level by binding with a variety of proteins that promote p53 degradation via the ubiquitin/ proteasome pathway (13). Among these p53 inhibitory proteins, $\mathrm{Mdm} 2$ is critically important for this process, since Mdm2-knockout mice show embryonic lethality but were fully rescued by deletion of p53 (34). When Cre-mediated conditional Mdm2-knockout mice were generated and studied, the findings revealed that $M d m 2$ deletion only in the central nervous system or the heart still led to embryonic lethality due to massive apoptosis, and this could also be rescued by deletion of $p 53(15,35)$. These findings demonstrated that Mdm2 functions as a crucial and specific $\mathrm{p} 53$ inhibitor in a variety of organs. In the present study, using hepatocyte-specific Mdm2-knockout mice, we could observe the consequences of persistent p53 activation in hepatocytes and discover the profibrotic function of $\mathrm{p} 53$.

Regarding the mechanism(s) involving spontaneous liver fibrosis in our Mdm2-knockout mice, we observed a mild increase in hepatocyte apoptosis (Figure 3, A-E). We have previously reported that hepatocyte-specific knockout of either Bcl-xL or Mcl-1, major antiapoptotic Bcl-2 family proteins, causes massive hepatocyte apoptosis and leads to liver fibrosis in mice $(16,36)$. Thus, although apoptosis is generally considered to be quiescent cell death that does not cause organ injury, hepatocyte apoptosis is apparently involved in liver fibrogenesis. However, in these mice, liver fibrosis is not evident at 6 weeks of age, although they show much higher ALT levels, more than $300 \mathrm{IU} / \mathrm{ml}$, and seem to have much more apoptosis than hepatocyte-specific Mdm2-knockout mice. Moreover, when hepatocyte apoptosis was similarly induced in Mdm2knockout mice and control littermates by administration of ABT-737, a Bcl-xL inhibitor that causes mild hepatocyte apoptosis in vivo (refs. 21, 22, and Supplemental Figure 11A), additional liver fibrogenic responses occurred in the knockout mice but not in the control littermates (Supplemental Figure 11B). Based on these findings, hepatocyte apoptosis could contribute to liver fibrosis, but some additional factors should be required for the liver fibrosis observed in the Mdm2-knockout mice.

In Mdm2-knockout mice, we also found an upregulation of CTGF synthesis in hepatocytes (Figure 7, A and B). CTGF, also known as CCN family 2 (CCN2), is one of the CCN family proteins and plays a pivotal role in fibrosis in the lung, skin, kidney, and heart (37) through extracellular matrix production, cell cycle control, and cell adhesion and migration $(14,38,39)$. With respect to the liver, several publications have reported that CTGF expression increases in human chronic liver fibrotic diseases such as chronic hepatitis C, NASH, and liver cirrhosis (40-43). Moreover, previous studies have shown that CTGF inhibition by siRNA had a beneficial effect on experimental liver fibrosis $(44,45)$, indicating that CTGF functions as an important profibrogenic cytokine in the liver. Although the main source of CTGF was thought to be HSCs and fibroblasts (46), recent reports have revealed that CTGF is also produced from hepatocytes $(47,48)$. Furthermore, transgenic mice expressing CTGF under the control of the albumin gene promoter showed exacerbation of liver fibrosis induced by chronic $\mathrm{CCl}_{4}$ administration (49), demonstrating that hepatocytederived CTGF plays an important role in liver fibrogenesis. However, Tong et al. (49) have also reported that hepatocyte-specific CTGF transgenic mice did not show spontaneous fibrosis in the liver without any fibrotic stimuli. Taken together, these findings support the idea that CTGF produced from hepatocytes may be an important factor for promoting liver fibrosis in the presence of apoptotic stimuli in Mdm2-knockout mice.

To further examine this idea, we performed an in vitro study using murine HSCs cocultured with apoptotic bodies prepared from hepatocytes. In agreement with a previous report (50), hepatocyte-derived apoptotic bodies efficiently activated HSCs (Supplemental Figure 12), and CTGF administration significantly upregulated type I collagen synthesis in HSCs in combination with apoptotic bodies (Supplemental Figure 13). Based on these results and in vivo findings, we suggest that hepatocyte p53 activation increased hepatocyte apoptosis and CTGF synthesis, and both together may induce HSC activation and collagen synthesis, contributing to the development of spontaneous liver fibrosis in Mdm2-knockout mice. It should be noted here that $\mathrm{p} 53$ activation did not appear to be a single causal factor for inducing apoptosis in two independent models of murine liver fibrosis but was a required for CTGF expression. Since CTGF expression was well correlated with p53 activation and liver fibrosis in human livers, p53-mediated CTGF induction may be a novel and important pathway for promoting liver fibrosis.

The Alb-Cre transgenic mice expressed cre recombinase in intrahepatic cholangiocytes as well as hepatocytes, as observed from $\beta$-galactosidase staining of the liver sections in Alb-Cre and Rosa26-LacZ double-transgenic mice (data not shown). In the present study, Mdm2-knockout mice ( $M d m 2^{f / f l}$ alb-cre) actually showed p53 accumulation and CTGF expression in some cholangiocytes (Supplemental Figure 14 and Figure 7B), and nutlin-3a treatment upregulated CTGF gene expression as well as p53-regulated genes in SNU-1079 cells, a human cholangiocellular carcinoma cell line with wild-type p53 status (ref. 51 and Supplemental Figure 15), suggesting the existence of the p53/CTGF pathway in cholangiocytes as well. However, CTGF expression was observed even in the cholangiocytes of control littermates, and its levels were not much different from those of the knockout mice (Figure 7B). Therefore, although cholangiocytes may contribute to hepatic CTGF synthesis in physiological settings, they may contribute less to the hepatic CTGF increase observed in Mdm2-knockout mice.

Recent research has shown that the CTGF gene is repressed by several miRNAs such as miR-18a, miR-30, and miR-133 (31, $32,52,53)$. In addition, a previous report has revealed that p53 represses $m i R-17-92$ transcription by binding to the p53-binding 
site overlapping the TATA box in the miR-17-92 promoter lesion (54). Thus, we focused on the miR-17-92 cluster (which includes miR-18) and identified a what we believe to be a novel regulatory mechanism by which p53 upregulates CTGF through repression of the miR-17-92 cluster gene in hepatocytes, revealing the involvement of this mechanism not only in vitro, but also in rodents and fibrotic human liver.

In the present study, we demonstrated a direct causal link between p53 activation in hepatocytes and liver fibrosis, as evidenced by the spontaneous liver fibrosis of hepatocyte-specific Mdm2-knockout mice and the alleviation of diet- or TAAinduced liver fibrosis in hepatocyte-specific p53-knockout mice. In hepatocytes, p53 activation induced the expression of CTGF, a key regulator of liver fibrosis, through miRNA regulation. Analysis of human tissues provided evidence that the p53/CTGF axis may be involved in human liver fibrosis. Recently, therapeutic applications of a 553 inhibitor have been proposed for preventing radiation-induced adverse events that are mediated by p53 activation (55). CTGF was also reported to increase in a variety of tissues, such as the liver, intestine, and colon, upon irradiation $(56,57)$ and play an important role in the progression of radiation-induced fibrosis $(57,58)$. Our present study suggests the possibility that positive regulation of CTGF by $\mathrm{p} 53$ may be a therapeutic target of organ fibrosis caused by irradiation therapy as well as disease.

\section{Methods}

Cell lines and materials. Human hepatoma cell line HepG2 and murine normal hepatocyte cell line BNL CL.2 were obtained from ATCC, and human cholangiocellular carcinoma cell line SNU-1079 was obtained from the Korean Cell Line Bank (51). They were cultured at $37^{\circ} \mathrm{C}$ under $5 \% \mathrm{CO}_{2}$ in DMEM containing 10\% FCS (Sigma-Aldrich). Nutlin-3a and Adriamycin were purchased from Sigma-Aldrich.

Human samples. Non-tumorous liver samples were obtained from 21 patients at surgical operation for hepatocellular carcinoma (HCC) $(n=10)$ and colorectal liver metastasis $(n=11)$. Among the 10 patients with HCC, 7 were positive for $\mathrm{HCV}$ antibody. Of the 10 patients, 4 were histologically diagnosed as having liver cirrhosis and 6 as having chronic hepatitis. The 11 metastatic patients were seronegative for both HBsAg and HCV antibodies. They all had normal liver function and were histologically diagnosed as non-fibrotic livers. The resected non-tumorous liver specimens were taken as far from the tumor as possible, soaked in RNAlater solution (Ambion), and then stored at $-80^{\circ} \mathrm{C}$ until use. Written informed consent was obtained from all patients according to a protocol approved by the Institutional Research Board of Osaka University Hospital.

Mice. Mdm $2^{f l / f l}$ mice were provided by Guillermina Lozano (University of Texas MD Anderson Cancer Center, Houston, Texas, USA) (15). Hepatocyte-specific Mdm2-knockout mice ( $M d m 2^{f / f l}$ alb-cre) were generated by mating of $M d m 2^{f l / f l}$ mice and Alb-Cre transgenic mice $(16,21)$. Trp53/ffl mice and ROSA26-LacZ mice were purchased from The Jackson Laboratory. Hepatocyte-specific Trp53-knockout mice (Trp5 $5 f^{f / f l}$ alb-cre) were generated by mating $\operatorname{Tr} p 53^{f / f l}$ mice and alb-cre transgenic mice. Hepatocytespecific Mdm2- and Trp53-double-knockout mice (Mdm $2^{f / f l} \operatorname{Trp} 53^{f / f l}$ alb-cre) were generated by mating $M d m 2^{f / f l} \operatorname{Tr} p 53^{f /+}$ mice and $M d m 2^{f / / f l} \operatorname{Tr} p 53^{f /+}$ alb-cre mice. Genomic recombination of the $M d m 2$ and $\operatorname{Tr} p 53$ genes occurred at a rate of about $75 \%$ in the entire liver (data not shown). C57BL/6J mice and BALB/c mice were purchased from Charles River Laboratories Japan. They were maintained in a specific pathogen-free facility and treated with humane care with approval from the Animal Care and Use Committee of Osaka University Medical School.
Isolation and culture of murine hepatocytes and NPCs. Hepatocytes and NPCs were isolated from $M d m 2^{f / f l}$ mice and $M d m 2^{f / f l}$ alb-cre mice by 2 -step collagenase-pronase perfusion of mouse livers as previously described (17). Isolated hepatocytes were maintained at $37^{\circ} \mathrm{C}$ under $5 \% \mathrm{CO}_{2}$ in William's Eagle medium containing 10\% FCS (Sigma-Aldrich), $100 \mathrm{nM}$ dexamethasone, $100 \mathrm{nM}$ insulin (Sigma-Aldrich) and L-glutamine (Invitrogen).

Histological analyses. The liver sections were stained with H\&E. For detection of apoptotic cells, the liver sections were also subjected to TUNEL staining, according to a previously reported procedure (36). To assess their regenerative status, we stained liver sections for nuclear BrdU incorporation as previously described (59). To assess fibrosis, we stained the liver sections with picrosirius red. The percentage of collagen deposition was quantified using image analysis software (WinROOF visual system, Mitani Corp.) (59). For immunohistochemical detection of p $53, \alpha$-SMA, cleaved caspase- 3 , and CTGF, the liver sections were respectively incubated with polyclonal rabbit anti-p53 antibody (Vector Laboratories Inc.), polyclonal rabbit anti- $\alpha$-SMA antibody (Santa Cruz Biotechnology Inc.), polyclonal rabbit anti-cleaved caspase-3 antibody (Cell Signaling Technology Inc.), and polyclonal goat anti-CTGF antibody (Santa Cruz Biotechnology Inc.).

Senescence-associated $\beta$-galactosidase assay. To assess hepatocyte senescence, we performed a senescence-associated $\beta$-galactosidase assay according to a previously described procedure (60). Briefly, the frozen liver sections were fixed in $0.25 \%$ glutaraldehyde for 10 minutes and immersed overnight in SA- $\beta$-gal staining solution $(0.5 \mathrm{mg} / \mathrm{ml} \mathrm{X-gal,} 3 \mathrm{mM}$ potassium ferricyanide, $3 \mathrm{mM}$ potassium ferrocyanide, $2 \mathrm{mM} \mathrm{MgCl} 2,0.25 \%$ Triton X-100, $0.1 \mathrm{M}$ phosphate buffer, $\mathrm{pH}$ 6.0).

Determination of liver bydroxyproline content. Hydroxyproline content was determined essentially as described previously (59). Results are expressed as micrograms of hydroxyproline per gram of wet liver.

Western blot analysis. Liver tissue was lysed in lysis buffer (1\% Nonidet P- $40,0.5 \%$ sodium deoxycholate, $0.1 \%$ sodium dodecyl sulfate, $1 \times$ protease inhibitor cocktail [Nacalai tesque], $1 \times$ phosphatase inhibitor cocktail [Nacalai tesque], phosphate-buffered saline, $\mathrm{pH}$ 7.4). The liver lysates were cleared by centrifugation at $10,000 \mathrm{~g}$ at $4^{\circ} \mathrm{C}$ for 15 minutes. The protein concentrations were determined using a bicinchoninic acid protein assay kit (Pierce). Equal amounts of protein lysates were electrophoretically separated by SDS polyacrylamide gels and transferred onto a polyvinylidene fluoride membrane. For immunodetection, the following antibodies were used: rabbit monoclonal antibody to $\mathrm{p} 53$, rabbit polyclonal antibody to Bax (Cell Signaling Technology), rabbit polyclonal antibody to $\mathrm{p} 21$, goat polyclonal antibody to CTGF, rabbit polyclonal antibody to p53 (Santa Cruz Biotechnology Inc.), rabbit polyclonal antibody to Noxa and p21 (Abcam), rabbit polyclonal antibody to Puma (ProSci Inc.), and mouse monoclonal antibody to $\beta$-actin (Sigma-Aldrich).

Real-time RT-PC for mRNA. Total RNA extracted from cell lines and liver tissues using the RNeasy Mini Kit (QIAGEN) was reverse transcribed and subjected to real-time RT-PCR as previously described (59). mRNA expression of the specific genes was quantified using TaqMan Gene Expression Assays (Applied Biosystems) as follows: murine Col1a1 (assay ID: Mm00801666_g1), murine Col1a2 (Mm01165187_m1), murine Ctgf (Mm01192933_g1), murine Pmaip1 (Mm00451763_m1), murine Cdkn1a (Mm01303209_m1), murine Bax (Mm0043205_m1), murine Trp53 (Mm01731287_m1), murine Tgfb1 (Mm01178820_m1), murine Pdgfb (Mm01298578_m1), murine Mmp2 (Mm00439506_m1), murine Mmp14 (Mm01318969_g1), murine Timp1 (Mm01341361_m1), murine Acta2 (Mm01546133_m1), murine miR-17-92 (Mm03307063_pri), murine Actb (Mm02619580_g1), human COL1A1 (Hs01076777_m1), human CTGF (Hs00170014_m1), human PMAIP1 (Hs00560402_m1), human CDKN1A (Hs00355782_m1), human TP53 (Hs01034249_m1), human TGFB1 (Hs00998133_m1), human PDGFB (Hs00234042_m1), human BBC3 (Hs00248075_m1), human 
BAX (Hs00180269_m1), and human ACTB (Hs99999903_m1). Human pri-miR-17-92 expression was quantified using the primers described previously (54). Transcript levels are presented as fold change.

Caspase-3/7 activity. Serum caspase-3/7 activity was measured with a luminescence substrate assay for caspase- 3 and caspase-7 (Caspase-Glo assay, Promega) as described previously (61).

Real-time RT-PCR assays for mature miRNA. Total RNA including the miRNA fraction extracted from cell lines using the miRNeasy Mini Kit (QIAGEN) was reverse transcribed. Quantitative PCR was performed with TaqMan MicroRNA Assays (Applied Biosystems) specific for miR-18a (assay ID: 002422), miR-19a (assay ID: 000395), and miR-19b (assay ID: 000396). To normalize the expression levels of miRNAs, we used the TaqMan MicroRNA Assay specific for RNU6B (assay ID: 001093) as the endogenous control. Transcript levels are presented as fold change.

Transfections with miRNA. HepG2 cells were transfected with $10 \mathrm{nM}$ PremiR miRNA precursor molecules (Ambion) of MIR18A (PM12973), MIR19A (PM10649), and MIR19B (PM10629) using RNAiMAX (Invitrogen) according to the manufacturer's instructions. Pre-miR negative control (Ambion) was used as a control.

Transfections with antisense of miRNA. HepG2 cells were transfected with $100 \mathrm{nM}$ Anti-miR miRNA inhibitor (Ambion) of MIR18A (AM12973), MIR19A (AM10649) and MIR19B (AM10629) using RNAiMAX (Invitrogen) according to the manufacturer's instructions. Anti-miR negative control (Ambion) was used as a control.

Dual luciferase reporter assay. Plasmid pTS-589, which contains the CTGF promoter linked to the upstream of a firefly luciferase reporter gene (29), was transfected into HepG2 cells together with pRL-TK (Promega) using Lipofectamine LTX (Invitrogen). Upon 24 hours of Nutlin-3a $(20 \mu \mathrm{M})$ or recombinant TGF- $\beta(10 \mathrm{ng} / \mathrm{ml})$ treatment, firefly luciferase activity was measured using the Luciferase Assay System (Promega) and normalized to Renilla luciferase activity.

siRNA-mediated knockdown. HepG2 cells were transfected with siRNA against TP53 (Validated Stealth RNAi siRNA, oligo ID: VHS40367) (Invitrogen) using Lipofectamine RNAi-MAX (Invitrogen) according to the manufacturer's protocol. Stealth RNAi Negative Control Low GC (Invitrogen) was used as the control.

Isolation and culture of mouse HSCs. HSCs were isolated from C57BL/6J mice by 2 -step collagenase-pronase perfusion of mouse liver, followed by density gradient centrifugation with $8.2 \%$ Nycodenz (Sigma-Aldrich) as previously described (59). Isolated $\mathrm{HSCs}$ were maintained at $37^{\circ} \mathrm{C}$ under $5 \% \mathrm{CO}_{2}$ in DMEM containing 10\% FCS (Sigma-Aldrich). HSCs after a few passages were used for the experiments.

Generation of apoptotic body and coculture experiment with HSCs. Apoptotic bodies of hepatocytes were generated as described previously (50). Briefly, BNL CL. 2 cells were UV irradiated $\left(100 \mathrm{~mJ} / \mathrm{cm}^{2}\right)$ and incubated for 2 days in DMEM with $10 \%$ FCS. Next, floating apoptotic bodies were collected and centrifuged at $1,500 \mathrm{~g}$ for 5 minutes. HSCs $\left(1.0 \times 10^{5}\right)$ were starved for 48 hours in DMEM without FCS and then cocultured with apoptotic bodies $\left(4.0 \times 10^{5}\right)$ in DMEM with $10 \%$ FCS for the indicated time courses with or without $100 \mathrm{ng} / \mathrm{ml}$ recombinant CTGF (EMP Genetech).

Experimental protocol for ABT-737 administration. ABT-737, provided by Abbott Laboratories, was dissolved with a mixture of $30 \%$ propylene glycol, $5 \%$ Tween 80 , and $65 \% \mathrm{D} 5 \mathrm{~W}$ ( $5 \%$ dextrose in water), to a final $\mathrm{pH}$ of $4-5$ as described previously (21). ABT-737 (100 mg/kg) was intraperitoneally administered to C57BL/6J mice, and 2 days later they were sacrificed for the various analyses.

Experimental protocol for ATH diet feeding. C57BL/6J mice, Trp53 $3^{f / / f l}$ mice, and $\operatorname{Trp} 53^{f / f l} /$ alb-cre mice were subdivided into two groups: (a) mice fed an ATH diet $(1.25 \%[\mathrm{w} / \mathrm{w}]$ cholesterol, $0.5 \%[\mathrm{w} / \mathrm{w}]$ cholic acid, and $16 \%[\mathrm{w} / \mathrm{w}]$ fat) for 4 weeks and (b) mice given standard chow (CRF-1, Charles River Laboratories Japan) for 4 weeks. After having been fasted for 8 hours, the animals were sacrificed for the various analyses.

Experimental protocol for TAA intraperitoneal administration. TAA $(200 \mathrm{mg} / \mathrm{kg})$ (Sigma-Aldrich) was intraperitoneally administered to $\operatorname{Tr} p 53^{\ell / f l}$ mice and

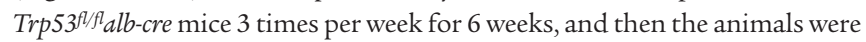
sacrificed for the various analyses.

Experimental protocol for hydrodynamic injection of $p 53$ expression plasmid. $\mathrm{BALB} / \mathrm{c}$ mice were given injection of pORF9-mp53 plasmid, an expression vector containing the murine p53 open reading frame (Invivogen) or its control plasmid via the tail vein by a hydrodynamic injection procedure (28) and sacrificed 2 days later for the various analyses.

Statistics. Data are expressed as median and interquartile range or mean $\pm \mathrm{SD}$. Statistical analyses were performed by the unpaired Mann-Whitney $U$ test or 1-way ANOVA unless otherwise indicated. When ANOVA analyses were applied, differences in the mean values among the groups were examined by Scheffe post hoc correction. Correlations were assessed using the Pearson product-moment correlation coefficient. $P$ values less than 0.05 were considered statistically significant.

\section{Acknowledgments}

We thank Guillermina Lozano (University of Texas MD Anderson Cancer Center) for providing the floxed $M d m 2$ mice. We also thank Abbott Laboratories for providing ABT-737. We thank Kanako Mori for help with experiments.

Received for publication August 31, 2010, and accepted in revised form May 12, 2011.

Address correspondence to: Tetsuo Takehara, Department of Gastroenterology and Hepatology, Osaka University Graduate School of Medicine, 2-2 Yamada-oka, Suita, Osaka 565-0871, Japan. Phone: 81.6.6879.3621; Fax: 81.6.6879.3629; E-mail: takehara@ gh.med.osaka-u.ac.jp.
1. Vousden $\mathrm{KH}, \mathrm{Lu} \mathrm{X}$. Live or let die: the cell's response to p53. Nat Rev Cancer. 2002;2(8):594-604.

2. Bensaad K, et al. TIGAR, a p53-inducible regulator of glycolysis and apoptosis. Cell. 2006;126(1):107-120.

3. Hu W, Zhang C, Wu R, Sun Y, Levine A, Feng Z. Glutaminase 2, a novel $\mathrm{p} 53$ target gene regulating energy metabolism and antioxidant function. Proc Natl Acad Sci U S A. 2010;107(16):7455-7460.

4. Crighton D, et al. DRAM, a p53-induced modulator of autophagy, is critical for apoptosis. Cell. 2006;126(1):121-134

5. Tyner SD, et al. p53 mutant mice that display early ageing-associated phenotypes. Nature. 2002; 415(6867):45-53.

6. Minamino T, et al. A crucial role for adipose tissue p53 in the regulation of insulin resistance. Nat Med.2009; 15(9):1082-1087.
7. Sano M, et al. p53-induced inhibition of Hif-1 causes cardiac dysfunction during pressure overload. Nature. 2007;446(7134):444-448

8. Akyol G, et al. P53 and proliferating cell nuclear antigen (PCNA) expression in non-tumoral liver diseases. Pathol Int. 1999;49(3):214-221.

9. Panasiuk A, Dzieciol J, Panasiuk B, Prokopowicz D. Expression of p53, Bax and Bcl-2 proteins in hepatocytes in non-alcoholic fatty liver disease. World J Gastroenterol. 2006;12(38):6198-6202.

10. Attallah AM, Shiha GE, Ismail H, Mansy SE, ElSherbiny R, El-Dosoky I. Expression of p53 protein in liver and sera of patients with liver fibrosis, liver cirrhosis or hepatocellular carcinoma associated with chronic HCV infection. Clin Biochem. 2009;42(6):455-461.

11. Loguercio C, et al. Liver p53 expression in patients with HCV-related chronic hepatitis. J Viral Hepat. 2003;10(4):266-270.

12. Papakyriakou P, et al. Apoptosis and apoptosis related proteins in chronic viral liver disease. Apoptosis. 2002;7(2):133-141.

13. Kruse JP, Gu W. Modes of p53 regulation. Cell. 2009;137(4):609-622.

14. Gressner OA, Gressner AM. Connective tissue growth factor: a fibrogenic master switch in fibrotic liver diseases. Liver Int. 2008;28(8):1065-1079.

15. Grier JD, Xiong S, Elizondo-Fraire AC, Parant JM, Lozano G. Tissue-specific differences of p53 inhibition by $\mathrm{Mdm} 2$ and Mdm4. Mol Cell Biol. 2006;26(1):192-198.

16. Takehara T, et al. Hepatocyte-specific disruption of Bcl-xL leads to continuous hepatocyte apoptosis and liver fibrotic responses. Gastroenterology. 
2004;127(4):1189-1197.

17. Sakamori R, et al. Signal transducer and activator of transcription 3 signaling within hepatocytes attenuates systemic inflammatory response and lethality in septic mice. Hepatology. 2007;46(5):1564-1573.

18. Vassilev LT, et al. In vivo activation of the p53 pathway by small-molecule antagonists of MDM2 Science. 2004;303(5659):844-848.

19. Bataller R, Brenner DA. Liver fibrosis. J Clin Invest. 2005;115(2):209-218.

20. Friedman SL. Mechanisms of hepatic fibrogenesis. Gastroenterology. 2008;134(6):1655-1669.

21 . Hikita $\mathrm{H}$, et al. $\mathrm{BH} 3$-only protein bid participates in the Bcl-2 network in healthy liver cells. Hepatology. 2009;50(6):1972-1980.

22. Hikita $\mathrm{H}$, et al. The Bcl-xL inhibitor, ABT-737, efficiently induces apoptosis and suppresses growth of hepatoma cells in combination with sorafenib. Hepatology. 2010;52(4):1310-1321.

23. Mitchell C, Willenbring $H$. A reproducible and well-tolerated method for $2 / 3$ partial hepatectomy in mice. Nat Protoc. 2008;3(7):1167-1170.

24. Larter CZ, Yeh MM. Animal models of NASH: getting both pathology and metabolic context right. J Gastroenterol Hepatol. 2008;23(11):1635-1648.

25. Matsuzawa $\mathrm{N}$, et al. Lipid-induced oxidative stress causes steatohepatitis in mice fed an atherogenic diet. Hepatology. 2007;46(5):1392-1403.

26 . Safadi R, et al. Immune stimulation of hepatic fibrogenesis by CD 8 cells and attenuation by transgenic interleukin-10 from hepatocytes. Gastroenterology. 2004;127(3):870-882.

27. Lin Y, et al. Tumour suppressor p53 and Rb genes in human hepatocellular carcinoma. Ann Acad Med Singapore. 1996;25(1):22-30.

28. Suzuki T, et al. Intravenous injection of naked plasmid DNA encoding hepatitis B virus (HBV) produces HBV and induces humoral immune response in mice. Biochem Biophys Res Commun. 2003 300(3):784-788.

29. Eguchi T, et al. Regulatory mechanism of human connective tissue growth factor (CTGF/Hcs24) gene expression in a human chondrocytic cell line, HCS-2/8. J Biochem. 2001;130(1):79-87.

30. Cicha I, Goppelt-Struebe M. Connective tissue growth factor: context-dependent functions and mechanisms of regulation. Biofactors. 2009; 35(2):200-208

31. Dews M, et al. Augmentation of tumor angiogenesis by a Myc-activated microRNA cluster. Nat Genet. 2006; 38(9):1060-1065

32. Ernst A, et al. De-repression of CTGF via the miR-17-92 cluster upon differentiation of human glioblastoma spheroid cultures. Oncogene. 2010 29(23):3411-3422.
33. Tanzer A, Stadler PF. Molecular evolution of a microRNA cluster. J Mol Biol. 2004;339(2):327-335.

34. Montes de Oca Luna R, Wagner DS, Lozano G. Rescue of early embryonic lethality in $\mathrm{mdm} 2$ deficient mice by deletion of p53. Nature. 1995; 378(6553):203-206

35. Xiong S, Van Pelt CS, Elizondo-Fraire AC, Liu G, Loz ano G. Synergistic roles of Mdm2 and Mdm4 for $\mathrm{p} 53$ inhibition in central nervous system development. Proc Natl Acad Sci US A. 2006;103(9):3226-3231.

36. Hikita $\mathrm{H}$, et al. Mcl-1 and $\mathrm{Bcl}-\mathrm{xL}$ cooperatively maintain integrity of hepatocytes in developing and adult murine liver. Hepatology. 2009;50(4):1217-1226.

37. Brigstock DR. The connective tissue growth factor/ cysteine-rich 61/nephroblastoma overexpressed (CCN) family. Endocr Rev. 1999;20(2):189-206.

38. Gao R, Brigstock DR. A novel integrin alpha5beta1 binding domain in module 4 of connective tissue growth factor (CCN2/CTGF) promotes adhesion and migration of activated pancreatic stellate cells. Gut. 2006;55(6):856-862.

39. Song JJ, et al. Connective tissue growth factor (CTGF) acts as a downstream mediator of TGFbeta 1 to induce mesenchymal cell condensation. J Cell Physiol. 2007;210(2):398-410.

40. Abou-Shady M, et al. Connective tissue growth factor in human liver cirrhosis. Liver. 2000;20(4):296-304.

41. Hora $\mathrm{C}$, et al. Connective tissue growth factor, steatosis and fibrosis in patients with chronic hepatitis C. Liver Int. 2008;28(3):370-376.

42. Williams EJ, Gaca MD, Brigstock DR, Arthur MJ, Benyon RC. Increased expression of connective tissue growth factor in fibrotic human liver and in activated hepatic stellate cells. J Hepatol. 2000;32(5):754-761.

43. Paradis V, et al. High glucose and hyperinsulinemia stimulate connective tissue growth factor expression: a potential mechanism involved in progression to fibrosis in nonalcoholic steatohepatitis. Hepatology. 2001;34(4 pt 1):738-744.

44. George J, Tsutsumi M. siRNA-mediated knockdown of connective tissue growth factor prevents $\mathrm{N}$-nitrosodimethylamine-induced hepatic fibrosis in rats. Gene Ther. 2007;14(10):790-803.

45. Li G, et al. Inhibition of connective tissue growth factor by siRNA prevents liver fibrosis in rats.J Gene Med. 2006;8(7):889-900.

46. Paradis V, et al. Expression of connective tissue growth factor in experimental rat and human liver fibrosis. Hepatology. 1999;30(4):968-976.

47. Gressner OA, Lahme B, Demirci I, Gressner AM, Weiskirchen R. Differential effects of TGF-beta on connective tissue growth factor (CTGF/CCN2) expression in hepatic stellate cells and hepatocytes. J Hepatol. 2007;47(5):699-710.
48. Weng HL, et al. Profibrogenic transforming growth factor-beta/activin receptor-like kinase 5 signaling via connective tissue growth factor expression in hepatocytes. Hepatology. 2007;46(4):1257-1270.

49. Tong Z, Chen R, Alt DS, Kemper S, Perbal B, Brigstock DR. Susceptibility to liver fibrosis in mice expressing a connective tissue growth factor transgene in hepatocytes. Hepatology. 2009;50(3):939-947.

50. Canbay A, Taimr P, Torok N, Higuchi H, Friedman S, Gores GJ. Apoptotic body engulfment by a human stellate cell line is profibrogenic. Lab Invest. 2003;83(5):655-663.

51. Ku JL, et al. Establishment and characterisation of six human biliary tract cancer cell lines. BrJ Cancer. 2002;87(2):187-193.

52. Ohgawara $\mathrm{T}$, et al. Regulation of chondrocytic phenotype by micro RNA 18a: involvement of Ccn2/Ctgf as a major target gene. FEBS Lett. 2009; 583(6):1006-1010.

53. Duisters RF, et al. miR-133 and miR-30 regulate connective tissue growth factor: implications for a role of microRNAs in myocardial matrix remodeling. Circ Res. 2009;104(2):170-178.

54. Yan HL, et al. Repression of the miR-17-92 cluster by p53 has an important function in hypoxia-induced apoptosis. EMBO J. 2009;28(18):2719-2732.

55. Komarov PG, et al. A chemical inhibitor of p53 that protects mice from the side effects of cancer therapy. Science. 1999;285(5434):1733-1737.

56. Ostrau C, et al. Lovastatin attenuates ionizing radiation-induced normal tissue damage in vivo. Radiother Oncol. 2009;92(3):492-499.

57. Vozenin-Brotons MC, et al. Fibrogenic signals in patients with radiation enteritis are associated with increased connective tissue growth factor expression. Int J Radiat Oncol Biol Phys. 2003;56(2):561-572.

58. Haydont V, Riser BL, Aigueperse J, Vozenin-Brotons MC. Specific signals involved in the longterm maintenance of radiation-induced fibrogenic differentiation: a role for CCN2 and low concentration of TGF-beta1. Am J Physiol Cell Physiol. 2008;294(6):C1332-C1341.

59. Kodama T, et al. Thrombocytopenia exacerbates cholestasis-induced liver fibrosis in mice. Gastroenterology. 2010;138(7):2487-2498.

60. Itahana K, Campisi J, Dimri GP. Methods to detect biomarkers of cellular senescence: the senescenceassociated beta-galactosidase assay. Methods $\mathrm{Mol}$ Biol. 2007;371:21-31.

61. Kodama T, et al. BH3-only activator proteins, Bid and Bim, are dispensable for Bak/Bax-dependent thrombocyte apoptosis induced by Bcl-xL deficiency: molecular requisites for the mitochondrial pathway to apoptosis in platelets. J Biol Chem. 2011;286(16):13905-13913 